\title{
Global higher integrability for minimisers of convex functionals with $(p, q)$-growth
}

\author{
Lukas Koch ${ }^{1}$
}

Received: 8 December 2020 / Accepted: 22 February 2021 / Published online: 3 April 2021

(c) The Author(s) 2021

\section{Abstract}

We prove global $W^{1, q}\left(\Omega, \mathbb{R}^{m}\right)$-regularity for minimisers of convex functionals of the form $\mathscr{F}(u)=\int_{\Omega} F(x, D u) \mathrm{d} x . W^{1, q}\left(\Omega, \mathbb{R}^{m}\right)$ regularity is also proven for minimisers of the associated relaxed functional. Our main assumptions on $F(x, z)$ are a uniform $\alpha$-Hölder continuity assumption in $x$ and controlled $(p, q)$-growth conditions in $z$ with $q<\frac{(n+\alpha) p}{n}$.

Mathematics Subject Classification Primary 49N60; Secondary 49N99 · 49J45

\section{Introduction and results}

We study minimisation problems of the form

$$
\min _{u \in W_{g}^{1, p}\left(\Omega, \mathbb{R}^{m}\right)} \mathscr{F}(u) \quad \text { where } \mathscr{F}(u)=\int_{\Omega} F(x, D u)-f \cdot u \mathrm{~d} x .
$$

Here $F(x, z)$ is a convex functional with $(p, q)$-growth in $z$ satisfying a natural uniform $\alpha$ Hölder condition in $x$. Furthermore $\Omega$ is a sufficiently regular domain in $\mathbb{R}^{n}, f \in L^{q^{\prime}}\left(\Omega, \mathbb{R}^{m}\right)$ and $g$ is a sufficiently regular boundary datum. We assume throughout that $1<p \leq q<$ $\infty$ and will impose further restrictions as necessary. For precise definitions we refer to Sect. 2 where we also explain our notation. We prove global higher integrability properties of the minimiser as well as global higher integrability properties of minimisers of a relaxed functional related to $\mathscr{F}(\cdot)$. Our results concern mostly the vectorial case $n, m \geq 2$. The one-dimensional case $n=1$ is special and stronger results apply.

The study of elliptic systems and functionals in the case where $p=q$ is well established with a very long list of important results. For an introduction and references we refer to [28] and [29].

In order to state our results precisely and to compare them with the literature on functionals with $(p, q)$-growth we briefly state our assumptions precisely.

Communicated by O. Savin.

$\bowtie$ Lukas Koch

kochl@maths.ox.ac.uk

1 University of Oxford, Andrew Wiles Building Woodstock Rd, Oxford OX2 6GG, UK 
Let $0<\alpha \leq 1$. We assume that $F(x, z)$ is measurable in $x$, continuously differentiable in $z$ and moreover satisfies

$$
\begin{gathered}
v\left(\mu^{2}+|z|^{2}+|w|^{2}\right)^{\frac{p-2}{2}} \leq \frac{F(x, z)-F(x, w)-\left\langle\partial_{z} F(x, w), z-w\right\rangle}{|z-w|^{2}} \\
|F(x, z)| \lesssim\left(1+|z|^{2}\right)^{\frac{q}{2}} \\
|F(x, z)-F(y, z)| \leq \Lambda|x-y|^{\alpha}\left(1+|z|^{2}\right)^{\frac{q}{2}} .
\end{gathered}
$$

for some $\mu, v, \Lambda>0$, all $z, w \in \mathbb{R}^{n \times m}$ and almost every $x, y \in \Omega$, where $1 \leq p \leq q$. If $p<n$ further suppose $q \leq \frac{n p}{n-p}$.

Note that under these assumptions $F(x, z)$ is convex. We also remark that our conditions clearly imply the following two bounds for $z \in \mathbb{R}^{n \times m}$ and almost every $x \in \Omega$,

$$
\begin{aligned}
|z|^{p}-1 & \lesssim F(x, z) \\
\left|\partial_{z} F(x, z)\right| & \lesssim \Lambda\left(1+|z|^{2}\right)^{\frac{q-1}{2}} .
\end{aligned}
$$

We refer to Sect. 6 for examples of functionals satisfying these assumptions. Alternative assumptions to (H1) that allow for anisotropic or $p(x)$-growth and under which the conclusions of Theorems 1 and 2 still hold are explored in Sect. 5.

Let us also give a precise definition of the notions of minimisers we are interested in:

Definition $1 u \in W_{g}^{1,1}(\Omega)$ is a (pointwise) minimiser of $\mathscr{F}(\cdot)$ in the class $W_{g}^{1, p}(\Omega)$ if it holds that $F(x, D u) \in L^{1}(\Omega)$ and

$$
\int_{\Omega} F(x, D u)-f \cdot u \mathrm{~d} x \leq \int_{\Omega} F(x, D u+D \phi)-f \cdot(u+\phi) \mathrm{d} x
$$

for all $\phi \in W_{0}^{1,1}(\Omega)$.

$u \in W_{g}^{1, p}(\Omega)$ is a $W^{1, q}$-relaxed minimiser (usually referred to as a relaxed minimiser) of $\mathscr{F}(\cdot)$ in the class $W_{g}^{1, p}(\Omega)$ if $u$ minimises the relaxed functional

$$
\overline{\mathscr{F}}(v)=\inf \left\{\liminf _{j \rightarrow \infty} \int_{\Omega} F\left(D v_{j}\right)-f \cdot v_{j}:\left(v_{j}\right) \subset Y, v_{j} \rightarrow v \text { weakly in } X\right\}
$$

amongst all $v \in X=W_{g}^{1, p}(\Omega)$ where $Y=W_{g}^{1, q}(\Omega)$.

Note that for a pointwise minimiser, $\mathscr{F}(u)<\infty$, coupled with (H1), Sobolev embedding and Young's inequality gives $D u \in L^{p}(\Omega)$. We remark further that by weak lower semicontinuity of $\mathscr{F}(\cdot)$ in $W^{1, q}(\Omega)$, for $u \in W_{g}^{1, q}(\Omega), \overline{\mathscr{F}}(u)=\mathscr{F}(u)$.

The study of regularity theory for minimisers in the case $p<q$ started with the seminal papers [36,37]. We don't aim to give a complete overview of the theory here, focusing on results directly relevant to this paper. We refer to [38] for a good overview and further references. A particular focus of research have been the special cases of the double-phase functional $F(x, z)=|z|^{p}+a(x)|z|^{q}$ and functionals with $p(x)$-growth. For an introduction and further references with regards to these special cases we refer to the introduction of [4] and $[17,41]$, respectively. Already in the scalar $m=1$ autonomous case $F(x, z)=F(z)$ counterexamples show that in order to prove regularity of minimisers $p$ and $q$ may not be too far apart $[27,32,36]$. We stress that the counterexamples only apply to pointwise minimisers (as opposed to relaxed minimisers, see Definition 3). A crucial first step to proving interior 
regularity results is to obtain $W_{\text {loc }}^{1, q}$ regularity of minimisers. Once $W_{\text {loc }}^{1, q}$ regularity is obtained, further regularity results such as partial regularity may be obtained, see for example [2,39]. We list the to our knowledge best available $W_{\text {loc }}^{1, q}$ regularity results for general autonomous convex functionals with $(p, q)$-growth (when $n \geq 2$ ): Under natural growth conditions it suffices to assume $q<\frac{n p}{n-1}$ [13] in order to obtain $W_{\text {loc }}^{1, q}$ regularity of minimisers. To obtain the same conclusion under controlled growth conditions the gap may be widened to $q<p\left(1+\frac{2}{n-1}\right)$ [44] and under controlled duality growth conditions it suffices to take $q<\frac{n p}{n-2}$ (if $n=2$ it suffices to take $q<\infty$ ) [15]. We note that in all three cases higher integrability goes hand in hand with a higher differentiability result. Partial $C^{1, \alpha}$ regularity of pointwise and relaxed minimisers (with $Y=W_{\text {loc }}^{1, q}$ ) for autonomous quasiconvex functionals has been obtained in [45-47] under natural growth conditions with $q<1+\frac{\min (2, p)}{2 n}$. The borderline-case $p=n-1, q=n$ was studied in [14]. Finally, we refer to [5] for results and references in the case of parabolic systems with $(p, q)$-growth.

We now turn to the case of non-autonomous functionals $F(x, z)$, convex and with $(p, q)$ growth in $z$, while satisfying a uniform $\alpha$-Hölder condition in $x$. For $n \geq 2$ counterexamples to $W^{1, q}$ regularity with $1<p<n<n+\alpha<q$ are due to [19], see also [24]. Recent work suggests that the condition $p<n<q$ may be removed [3]. If $q<\frac{(n+\alpha) p}{n}$, it was proven in [19] for many standard examples that minimisers enjoy $W_{\mathrm{loc}}^{1, q}$ regularity. Using [18] the result may be extended to functionals satisfying in addition the following condition:

there is $\varepsilon_{0}>0$ such that for any $\varepsilon \in\left(0, \varepsilon_{0}\right)$ and $x \in \Omega$ there exists $\hat{y} \in \overline{B_{\varepsilon}(x) \cap \Omega}$ such that

$$
F(\hat{y}, z) \leq F(y, z) \quad \forall y \in \overline{B_{\varepsilon}(x) \cap \Omega}, \quad z \in \mathbb{R}^{n \times m} .
$$

(H4) is very similar to Assumption 2.3. in [53]. We remark that (H4) holds for many of the commonly considered examples, see Sect. 6 as well as $[18,19] . W_{\text {loc }}^{1, q}$ regularity is in general not known if $q=\frac{(n+\alpha) p}{n}$. An exception are functionals modeled on the doublephase functional [4], see also [16].

One of the few results on regularity up to the boundary in the setting of $(p, q)$-growth functionals is [7] where Lipschitz regularity up to the boundary is obtained for minimisers of scalar autonomous functionals satisfying nonstandard growth conditions and the structure condition $F(x, z)=b(|z|)$. The growth conditions considered include $(p, q)$-growth. For functionals satisfying a structural assumption inspired by the double-phase functional Caldéron-Zygmund estimates valid up to the boundary are obtained in [10]. Hölder-regularity up to the boundary for double-phase functionals is studied in [48].

If additional structure assumptions such as $F(x, z)=b(x,|z|)$ are imposed or if it is assumed that minimisers are bounded it is possible to improve on the results listed so far. Without going into further detail we refer to $[6,12]$ for results and further references in these directions. Local boundedness of minimisers for convex non-autonomous $(p, q)$-growth functionals under natural growth conditions and the additional assumption $F(x, 2 z) \lesssim 1+$ $F(x, z)$ is studied in [31].

The main result of this paper is to obtain global $W^{1, q}$ regularity of minimisers for nonautonomous functionals $F(x, z)$ - convex and with controlled $(p, q)$-growth conditions in $z$ as well as satisfying a uniform $\alpha$-Hölder condition in $x$ - if $q<\frac{(n+\alpha) p}{n}$ and under the additional assumption (H4). Thus our results are the global analogue of the local results in $[18,19]$. We stress that we do not make any structure assumption on the dependence on $z$ going beyond controlled $(p, q)$-growth conditions and that we make no geometric assumptions on either 
the domain or the boundary datum $g$. To the best of our knowledge this is the first global $W^{1, q}$ regularity result valid for a large class of general convex $(p, q)$-growth functionals.

Proofs of $W_{\text {loc }}^{1, q}$-regularity in the $(p, q)$-growth framework often involve two major steps. First, an a-priori estimate for minimisers of a suitably regularised $q$-growth functional is obtained using difference quotient methods. Second, taking limits the estimate is transferred to minimisers of the original functional. In this paper we follow the same ideas. In order to apply the difference quotient method globally, we rely on an argument developed in [43]. Using this technique we obtain a regularity result for relaxed minimisers:

Theorem 1 Suppose $\Omega$ is a Lipschitz domain. Let $g \in W^{1+\alpha, q}(\Omega)$. Suppose $F(x, \cdot)$ satisfies (H1)-(H3) with $1<p \leq q<\frac{(n+\alpha) p}{n}$. If $u$ is a relaxed minimiser of $\mathscr{F}(\cdot)$ in the class $W_{g}^{1, p}(\Omega)$, then $u \in W^{1, q}(\Omega)$. Moreover for any $0 \leq \beta<\alpha$ there is $\gamma>0$ such that

$$
\|u\|_{W^{1, \frac{n p}{n-\beta}(\Omega)}} \lesssim\left(1+\overline{\mathscr{F}}(u)+\|g\|_{W^{1+\alpha, q}(\Omega)}+\|f\|_{L^{q^{\prime}(\Omega)}}\right)^{\gamma} .
$$

A key obstruction to $W^{1, q}$-regularity in the $(p, q)$-growth setting is the Lavrentiev phenomenon, which describes the possibility that

$$
\inf _{u \in W_{g}^{1, p}(\Omega)} \mathscr{F}(u)<\inf _{u \in W_{g}^{1, q}(\Omega)} \mathscr{F}(u) .
$$

A first example of this phenomenon was given in [35]. In the context of $(p, q)$ - growth functionals the theory was further developed in [51-53]. The Lavrentiev phenomenon is closely related to properties of the relaxed functional. We adopt the viewpoint and terminology of [9] and consider a topological space $X$ of weakly differentiable functions and a dense subspace $Y \subset X$. We introduce the following sequentially lower semi-continuous (slsc) envelopes

$$
\begin{aligned}
& \overline{\mathscr{F}}_{X}=\sup \{\mathscr{G}: X \rightarrow[0, \infty]: \mathscr{G} \text { slsc }, \mathscr{G} \leq \mathscr{F} \text { on } X\} \\
& \overline{\mathscr{F}}_{Y}=\sup \{\mathscr{G}: X \rightarrow[0, \infty]: \mathscr{G} \text { slsc }, \mathscr{G} \leq \mathscr{F} \text { on } Y\}
\end{aligned}
$$

and define the Lavrentiev gap functional for $u \in X$ as

$$
\mathscr{L}(u, X, Y)= \begin{cases}\overline{\mathscr{F}}_{Y}(u)-\overline{\mathscr{F}}_{X}(u) & \text { if } \overline{\mathscr{F}}_{X}(u)<\infty \\ 0 & \text { else. }\end{cases}
$$

Note that the gap functional is non-negative.

There is an extensive literature on the Lavrientiev phenomenon and gap functional, an overview of which can be found in $[8,25]$ to which we also refer for further references. The phenomenon is also of interest in nonlinear elasticity [26]. Considering the common choice $X=W^{1, p}(\Omega)$ endowed with the weak toplogy, $Y=W_{\text {loc }}^{1, q}(\Omega)$ a question related to the Lavrentiev phenomenon is to study measure representations of $\overline{\mathscr{F}}(\cdot)$. We refer to $[1,22]$ for results and further references in this direction, but point out that in this context in [23] an argument using a Whitney cover of $\Omega$ was given that is similar to arguments in this paper.

In this paper we always consider the choice $X=W_{g}^{1, p}(\Omega)$ endowed with the weak topology and $Y=W_{g}^{1, q}(\Omega)$. Since $F(x, z)$ is convex, standard methods show that then $\overline{\mathscr{F}}_{X}(\cdot)=$ $\mathscr{F}(\cdot)$, see [29, Chapter 4]. Further $\overline{\mathscr{F}}_{Y}(\cdot)=\overline{\mathscr{F}}(\cdot)$. We also note that if $\mathscr{L}(u, X, Y)=0$ for all $u \in X$, then the Lavrentiev phenomenon cannot occur. Non-occurrence of the Lavrentiev phenomenon allows to transfer the estimates obtained in Theorem 1 to pointwise minimisers and thus to establish $W^{1, q}$-regularity. In fact under the assumption that $\mathscr{L}(u, X, Y)=0$ 
for all $u \in X$ with $X=W^{1, p}(\Omega)$, endowed with the weak toplogy, and $Y=W^{1, p}\left(B_{r}\right)$ with $B_{r} \Subset \Omega, W^{1, q}\left(B_{r}\right)$-regularity of minimisers of non-autonomous functionals satisfying (H1)-(H3) with $1<p \leq q<\frac{(n+\alpha) p}{n}$ is obtained in [19]. Under (H1)-(H3) and the same restriction on $q$, [18] shows that $\mathscr{L}\left(\cdot, W^{1, p}(\Omega), W^{1, q}\left(B_{r}\right)\right)=0$ where $B_{r} \Subset \Omega$ if in addition (H4) holds. Our next theorem obtains global $W^{1, q}$-regularity of minimisers under the same assumptions.

Theorem 2 Suppose $\Omega$ is a $C^{1, \alpha}$-domain. Suppose $g \in W^{1+\max \left(\alpha, \frac{1}{q}\right), q}(\Omega)$. Assume $\mathscr{F}(\cdot)$ satisfies (H1)-(H3) with $1<p \leq q<\frac{(n+\alpha) p}{n}$ and (H4). Suppose $u$ is a pointwise minimiser of $\mathscr{F}(\cdot)$ in the class $W_{g}^{1, p}(\Omega)$. Then $u \in W^{1, q}(\Omega)$. Moreover for $0 \leq \beta<\alpha$ there is $\gamma>0$ such that

$$
\|u\|_{W^{1, \frac{n p}{n-\beta}(\Omega)}} \lesssim\left(1+\mathscr{F}(u)+\|g\|_{W^{1+\max \left(\alpha, \frac{1}{q}\right), q}(\Omega)}+\|f\|_{L^{q^{\prime}(\Omega)}}\right)^{\gamma}
$$

The proof of this theorem employs regular approximations to $u \in W_{g}^{1, p}(\Omega)$ using a partition of unity adapted to a Whitney-Besicovitch covering of $\Omega$. This construction is motivated by the following considerations: For autonomous convex functionals it is easy to see that considering the mollified functions $u \star \phi_{\varepsilon}$ shows that $\mathscr{L}(\cdot, X, Y)=0$ on $X=W^{1, p}(\Omega)$ where $Y=W^{1, q}(\omega)$ for some fixed $\omega \Subset \Omega$. Our situation, where we intend to employ a similar argument, differs in two aspects: we consider non-autonomous functionals and require results that are valid up to the boundary. We use assumption (H4) to deal with the $x$-dependence. In order to obtain results valid up to the boundary our main idea is to use a two-parameter mollification $u \star \phi_{\varepsilon \delta(x)}$ where $\delta(x) \sim d(x, \partial \Omega)$. We will implement this using a Whitney-Besicovitch covering.

We are not able to prove that $\mathscr{L}\left(u, W_{g}^{1, p}(\Omega), W_{g}^{1, q}(\Omega)\right)=0$ for all $u \in W_{g}^{1, p}(\Omega)$ in the full range of $q$ covered by Theorem 2 , but obtain the following partial result:

Proposition 1 Suppose $\Omega$ is a Lipschitz domain and $g \in W^{1+\frac{1}{q}, q}(\Omega)$. Let $X=W_{g}^{1, p}(\Omega)$ endowed with the weak topology. Suppose that in addition $1<p \leq q<\frac{(n+\alpha) p}{n}$ and that $F(x, \cdot)$ satisfies $(\mathrm{H} 1)-(\mathrm{H} 3)$ and $(\mathrm{H} 4)$. Then, with $Y=W_{\mathrm{loc}}^{1, q}(\Omega)$,

$$
\mathscr{L}(\cdot, X, Y)=0 \text { on } X .
$$

If in fact $1<p \leq q<\min \left(p+1,\left(1+\frac{\alpha}{(\alpha+1) n}\right) p\right)$, then $\mathscr{L}(\cdot, X, Y)=0$ on $X$ with the choice $Y=W_{g}^{1, q}(\Omega)$.

We mention that as a by-product of our work we also obtain a higher differentiability result:

Corollary 1 Suppose the assumptions of Theorem 1 (or Theorem 2) hold. Let $u$ be a relaxed (pointwise) minimiser of $\mathscr{F}(\cdot)$ in the class $W_{g}^{1, p}(\Omega)$. Then $u \in W^{1+\beta, p}(\Omega)$ for $\beta<\frac{\alpha}{\max (2, p)}$. Moreover there is $\gamma>0$ such that

$$
\|u\|_{W^{1+\beta, p}(\Omega)} \lesssim\left(1+\mathscr{F}(u)+\|g\|_{W^{1+\max \left(\alpha, \frac{1}{q}\right), q}(\Omega)}+\|f\|_{L^{q^{\prime}(\Omega)}}\right)^{\gamma}
$$

The structure of the paper is as follows: In Sect. 2 we collect some background results that we use to prove global higher integrability for minimisers of the relaxed functional in Sect. 3 and for minimisers of the pointwise functional in Sect. 4. In Sect. 5 we extend these results to ellipticity assumptions suited for anisotropic and $p(x)$-growth. In Sect. 6 we give examples of functionals to which our results apply. 


\section{Preliminaries}

\subsection{Notation}

In this section we introduce our notation. The set $\Omega$ will always denote a open, bounded domain in $\mathbb{R}^{n}$. Given a set $\omega \subset \mathbb{R}^{n}, \bar{\omega}$ will denote its closure. We write $B_{r}(x)$ for the usual open Euclidean balls in $\mathbb{R}^{n}$ and $S^{n-1}$ for the unit sphere in $\mathbb{R}^{n}$. We denote the cone of height $\rho$, aperture $\theta$ and axis in direction $\boldsymbol{n}$ by $C_{\rho}(\theta, \boldsymbol{n})$. That is

$$
C_{\rho}(\theta, \boldsymbol{n})=\left\{h \in \mathbb{R}^{n}:|h| \leq \rho, h \cdot \boldsymbol{n} \geq|h| \cos (\theta)\right\} .
$$

Here $|\cdot|$ denotes the Euclidean norm of a vector in $\mathbb{R}^{n}$ and likewise the Euclidean norm of a matrix $A \in \mathbb{R}^{n \times n}$. Id denotes the identity matrix in $\mathbb{R}^{n \times n}$. Given an open set $\Omega$ we denote $\Omega_{\lambda}=\{x \in \Omega: d(x, \partial \Omega)>\lambda\}$ and $\lambda \Omega=\{\lambda x: x \in \Omega\}$. Here $d(x, \partial \Omega)=$ $\inf _{y \in \partial \Omega}|x-y|$ denotes the distance of $x$ from the boundary of $\Omega$.

If $p \in[1, \infty]$ denote by $p^{\prime}=\frac{p}{p-1}$ its Hölder conjugate. The symbol $a \lesssim b$ means that there is some constant $C>0$, depending only on $n, m, p, \Omega, \mu, v$ and $\Lambda$, and independent of $a$ and $b$ such that $a \leq C b$.

Write $V_{\mu, t}(z)=\left(\mu^{2}+|z|^{2}\right)^{\frac{t-2}{4}} z$. We recall the useful well-known inequality:

Lemma 1 For $s>-1, \mu \in[0,1], z_{1}, z_{2} \in \mathbb{R}^{N}$, with $\mu+\left|z_{1}\right|+\left|z_{2}\right|>0$,

$$
\int_{0}^{1}\left(\mu^{2}+\mid z_{1}+\lambda\left(z_{2}-z_{1}\right)^{2}\right)^{\frac{s}{2}} \lambda \mathrm{d} \lambda \sim\left(\mu^{2}+\left|z_{1}\right|^{2}+\left|z_{2}\right|^{2}\right)^{\frac{s}{2}}
$$

with the implicit constants only depending on s. Further,

$$
\left|V_{\mu, t}\left(z_{1}\right)-V_{\mu, t}\left(z_{2}\right)\right| \sim\left(\mu^{2}+\left|z_{1}\right|^{2}+\left|z_{2}\right|^{2}\right)^{\frac{t-2}{2}}\left|z_{1}-z_{2}\right|^{2} .
$$

We will often find it useful to write for a function $v$ defined on $\mathbb{R}^{n}$ and a vector $h \in \mathbb{R}^{n}$, $v_{h}(x)=v(x+h)$.

We pick a family $\left\{\phi_{\varepsilon}\right\}$ of radially symmetric, non-negative mollifiers of unitary mass. We denote convolution with $\phi_{\varepsilon}$ as

$$
u \star \phi_{\varepsilon}(x)=\int_{\mathbb{R}^{n}} u(y) \phi_{\varepsilon}(x-y) \mathrm{d} y .
$$

\subsection{Function spaces}

We recall some basic properties of Sobolev and Besov spaces following the exposition in [43], alternatively the theory can be found in [49].

For $0 \leq \alpha \leq 1$ and $k \in \mathbb{N}, C^{k}(\Omega)$ and $C^{k, \alpha}(\Omega)$ denote the spaces of functions $k$-times continuously differentiable in $\Omega$ and $k$-times $\alpha$-Hölder differentiable in $\Omega$, respectively.

For $1 \leq p \leq \infty, k \in \mathbb{N}, L^{p}(\Omega)=L^{p}\left(\Omega, \mathbb{R}^{m}\right)$ and $W^{k, p}(\Omega)=W^{k, p}\left(\Omega, \mathbb{R}^{m}\right)$ denote the usual Lebesgue and Sobolev spaces respectively. We write $W_{0}^{k, p}(\Omega)$ for the closure of $C_{0}^{\infty}(\Omega)$-functions with respect to the $W^{k, p}$-norm. For functions $g \in W^{1,1}(\Omega)$, write $W_{g}^{k, p}(\Omega)=g+W_{0}^{k, p}(\Omega)$. We freely identify $W^{k, p}$-functions with their precise representatives.

Denote by $[,]_{s, q}$ the real interpolation functor. Let $s \in(0,1)$ and assume $p, q \in[1, \infty]$. We define

$$
B_{q}^{s, p}(\Omega)=B_{q}^{s, p}\left(\Omega, \mathbb{R}^{m}\right)=\left[W^{1, p}\left(\Omega, \mathbb{R}^{m}\right), L^{p}\left(\Omega, \mathbb{R}^{m}\right)\right]_{s, q}
$$




$$
B_{q}^{1+s, p}(\Omega)=\left[W^{2, p}(\Omega), W^{1, p}(\Omega)\right]_{s, q}=\left\{v \in W^{1, p}(\Omega): D v \in B_{q}^{s, p}(\Omega)\right\}
$$

Further we recall that $W^{1+s, p}(\Omega)=B_{p}^{1+s, p}(\Omega)$ and that for $1 \leq q<\infty, B_{q}^{s, p}(\Omega)$ embeds continuously in $B_{\infty}^{s, p}(\Omega)$. We will use a characterisation of these spaces in terms of difference quotients. Let $D$ be a set generating $\mathbb{R}^{n}$, star-shaped with respect to 0 . For $s \in(0,1)$, $p \in[1, \infty]$, consider

$$
[v]_{s, p, \Omega}^{p}=\sup _{h \in D \backslash\{0\}} \int_{\Omega_{h}}\left|\frac{v_{h}(x)-v(x)}{h}\right|^{p} \mathrm{~d} x .
$$

Here $v_{h}(x)=v(x+h)$. This characterises $B_{\infty}^{s, p}(\Omega)$ in the sense that

$$
v \in B_{\infty}^{s, p}(\Omega) \Leftrightarrow v \in L^{p}(\Omega) \text { and }[v]_{s, p, \Omega}^{p}<\infty .
$$

Moreover there are positive constants $C_{1}, C_{2}>0$ depending only on $s, p, D, \Omega$ such that

$$
C_{1}\|v\|_{B_{\infty}^{s, p}(\Omega)} \leq\|v\|_{L^{p}(\Omega)}+[v]_{s, p, \Omega} \leq C_{2}\|v\|_{B_{\infty}^{s, p}(\Omega)} .
$$

If $\Omega=B_{r}\left(x_{0}\right)$, then $C_{1}, C_{2}$ are unchanged by replacing $D$ with $Q D$, where $Q$ is an orthonormal matrix. In particular, when $D=C_{\rho}(\theta, \boldsymbol{n})$ is a cone, they are independent of the choice of $\boldsymbol{n}$.

Finally, recall that $B_{q}^{s, p}(\Omega)$ may be localised: If $\left\{U_{i}\right\}_{i \leq M}$ is a finite collection of balls covering $\Omega$, then $v \in B_{q}^{s, p}(\Omega)$ if and only if $v_{\mid \Omega \cap U_{i}} \in B_{q}^{s, p}\left(\Omega \cap U_{i}\right)$ for $i=1, \ldots, M$. Moreover, there are constants $C_{3}, C_{4}$ depending only on $M$ such that

$$
C_{3}\|v\|_{B_{q}^{s, p}(\Omega)} \leq \sum_{i=1}^{M}\|v\|_{B_{q}^{s, p}\left(\Omega \cap U_{i}\right)} \leq C_{4}\|v\|_{B_{q}^{s, p}(\Omega)} .
$$

We recall a well-known embedding theorem, see e.g. [50]:

Theorem 3 Let $0<s \leq 1$ and $p, p_{1} \in[1, \infty]$. Assume that $s-\frac{n}{p}=-\frac{n}{p_{1}}$ and suppose $v \in B_{\infty}^{s, p}(\Omega)$. Then for any $\varepsilon \in\left(0,1-p_{1}\right]$,

$$
\|v\|_{L^{p_{1}-\varepsilon}(\Omega)} \lesssim\|v\|_{B_{\infty}^{s, p}(\Omega)} .
$$

We next recall some basic properties of mollifications. Proofs can be found in [20].

Lemma 2 Let $1<p<\infty$. Let $\Omega$ be an open domain in $\mathbb{R}^{n}$ and $\omega \Subset \Omega$. Suppose $u \in$ $W^{1, p}(\Omega)$ and consider $u_{\varepsilon}=u \star \phi_{\varepsilon}$ for $\varepsilon<d(\omega, \partial \Omega)$. Then

(i) $u_{\varepsilon} \in C^{\infty}(\omega)$

(ii) $\left\|u_{\varepsilon}\right\|_{W^{1, p}(\omega)} \leq\|u\|_{W^{1, p}(\Omega)}$

(iii) For $p \leq q \leq \frac{n p}{n-p}$, $\left\|u_{\varepsilon}-u\right\|_{L^{p}(\omega)} \lesssim \varepsilon^{\Theta}\|u\|_{W^{1, p}(\Omega)}$ where $\Theta=1+n(1 / q-1 / p)$ if $p<n, \Theta=n / q$ if $p \geq n$.

(iv) $\left\|D u_{\varepsilon}\right\|_{L^{\infty}(\omega)} \lesssim \varepsilon^{-\frac{n}{p}}\|D u\|_{W^{1, p}(\Omega)}$.

We also recall the trace theorem in the following form, see e.g. [21].

Lemma 3 Let $\Omega$ be a Lipschitz domain. Let $1<p<\infty$. Then there is a bounded linear operator $\operatorname{Tr}: W^{1+\frac{1}{p}, p} \Omega \rightarrow W^{1, p}(\partial \Omega)$. In fact, it is possible to define $\operatorname{Tr} u$ to be the values of the precise representative of $u$ on $\partial \Omega$.

Finally we recall the following well-known result (see [21] for the ingredients of the proof) which will justify extending $u$ by extensions of $g$. 
Lemma 4 Let $p \in[1, \infty]$. Let $V \ni \Omega$ be an open, bounded set. Suppose $u \in W^{1, p}(\Omega)$ and $v \in\left(u+W_{0}^{1, p}(\Omega)\right) \cap W^{1, p}(V)$. Then the map

$$
w=\left\{\begin{array}{l}
u \text { in } \Omega \\
v \text { in } V \backslash \Omega
\end{array}\right.
$$

belongs to $W^{1, p}(V)$.

\subsection{Some properties of Lipschitz and $C^{1, \alpha}$ domains}

In this section we recall some properties of Lipschitz and $C^{1, \alpha}$-domains. For further details we refer to [30]. We say $\Omega \subset \mathbb{R}^{n}$ is a Lipschitz $\left(C^{1, \alpha}\right)$ domain if $\Omega$ is an open subset of $\mathbb{R}^{n}$ and for every $x \in \partial \Omega$, there exists a neighbourhood $V$ of $x$ in $\mathbb{R}^{n}$ and orthogonal coordinates $\left\{y_{i}\right\}_{1 \leq i \leq n}$ such that

(i) $V$ is a hypercube in the new coordinates:

$$
V=\left\{\left(y_{1}, \ldots, y_{n}\right\}:-a_{i}<y_{i}<a_{i}, 1 \leq i \leq n-1\right\} .
$$

(ii) there exists a Lipschitz $\left(C^{1, \alpha}\right)$ function $\phi$ defined in

$$
V^{\prime}=\left\{\left(y_{1}, \ldots y_{n-1}\right):-a_{i}<y_{i}<a_{i}, 1 \leq i \leq n-1\right\}
$$

and such that

$$
\begin{array}{r}
\left|\phi\left(y^{\prime}\right)\right| \leq a_{n} / 2 \text { for every } y^{\prime}=\left(y_{1}, \ldots, y_{n-1}\right) \in V^{\prime}, \\
\Omega \cap V=\left\{y=\left(y^{\prime}, y_{n}\right) \in V: y_{n}<\phi\left(y^{\prime}\right)\right\}, \\
\partial \Omega \cap V=\left\{y=\left(y^{\prime}, y_{n}\right) \in V: y_{n}=\phi\left(y^{\prime}\right)\right\} .
\end{array}
$$

Let $\Omega$ be a Lipschitz domain. Then $\Omega$ satisfies a uniform exterior cone condition [30, Section 1.2.2]: there are $\rho_{0}, \theta_{0}>0$ and a map $\boldsymbol{n}: \mathbb{R}^{n} \rightarrow S^{n-1}$ such that for every $x \in \mathbb{R}^{n}$

$$
C_{\rho_{0}}\left(\theta_{0}, \boldsymbol{n}(x)\right) \subset O_{\rho_{0}}(x)=\left\{h \in \mathbb{R}^{n}:|h| \leq \rho_{0},\left(B_{3 \rho_{0}}(x) \backslash \Omega\right)+h \subset \mathbb{R}^{n} \backslash \Omega\right\} .
$$

Moreover there is a smooth vector field transversal to $\partial \Omega$, i.e. there exists $\kappa>0$ and $X \in C^{\infty}\left(\mathbb{R}^{n}, \mathbb{R}^{n}\right)$ such that

$$
X \cdot v \geq \kappa
$$

a.e. on $\partial \Omega$ where $v$ is the exterior unit normal to $\partial \Omega$ [30, Lemma 1.5.1.9.].

It is well-known that every Lipschitz domain can be written as a finite union of strongly star-shaped Lipschitz domains:

Lemma 5 Suppose $\Omega$ is a Lipschitz domain. Then there is $N>0$ and strongly starshaped Lipschitz domains $\Omega_{i}, \omega_{i}, i=1, \ldots N$ such that $\omega_{i} \Subset \Omega_{i}$ relative to $\Omega, \partial \omega_{i} \cap \partial \Omega \Subset \partial \Omega_{i} \cap \partial \Omega$ if $\partial \Omega_{i} \cap \Omega \neq \emptyset$ and

$$
\Omega=\bigcup_{i=1}^{N} \Omega_{i}=\bigcup_{i=1}^{N} \omega_{i} .
$$

Moreover given $g \in W^{1+\frac{1}{q}, q}(\Omega)$ and $u \in W_{g}^{1, p}(\Omega)$, we can ensure that we have $u \in W^{1, p}\left(\partial \Omega_{i}\right)$ for $i=1, \ldots, N$ with

$$
\|u\|_{W^{1, p}\left(\partial \Omega_{i}\right)} \lesssim\|u\|_{W^{1, p}\left(\Omega_{i}\right)}+\|g\|_{W^{1+1 / q, q}(\Omega)} .
$$


Proof We recall the main points of the proof and refer to [11, Proposition 2.5.3] for details. Let $x \in \partial \Omega$. Then for $\varepsilon$ and $\rho$ sufficiently small we may assume that $I_{x}$ is a neighbourhood of $x$ such that, writing $\hat{y}=\left(y_{1}, \ldots, y_{n-1}\right)$,

$$
I_{x} \cap \bar{\Omega}=\left\{y \in \mathbb{R}^{n}:-\varepsilon<y_{n} \leq \tau(\hat{y}), \quad \hat{y} \in B_{\rho}(0)\right\}
$$

where $\tau$ is Lipschitz function. We may further assume that $\rho \leq \frac{\tau(0)}{2 c}$ where $c$ denotes the Lipschitz constant of $\tau$. We now consider

$$
\Omega_{x}^{\varepsilon^{\prime}, \rho^{\prime}}=\left\{y \in \mathbb{R}^{n}:-\varepsilon^{\prime}<y_{n}<\tau(\hat{y}), \quad \hat{y} \in B_{\rho^{\prime}}(0)\right\}
$$

with $\varepsilon^{\prime} \in(0, \varepsilon), \rho^{\prime} \in(0, \rho)$. A straightforward argument shows that $\Omega^{\varepsilon^{\prime}, \rho^{\prime}}$ is strongly star-shaped with respect to 0 as well as a Lipschitz domain. Since

$$
\int_{0}^{\varepsilon} \int_{0}^{\rho} \int_{\partial \Omega_{x}^{\varepsilon^{\prime}, \rho^{\prime}}}\left(|u|^{p}+|D u|^{p}\right) \lesssim \int_{I_{x} \cap \Omega}|D u|^{p} \mathrm{~d} x
$$

we can choose $\tilde{\varepsilon} \in(0, \varepsilon)$ and $\tilde{\rho} \in(0, \rho)$ such that

$$
\int_{\partial \Omega_{x}^{\varepsilon^{\prime}, \rho^{\prime}} \backslash \partial \Omega}|u|^{p}+|D u|^{p} \lesssim c(\varepsilon, \rho)\|u\|_{W^{1, p}(\Omega)} .
$$

We now set $\Omega_{x}=\Omega_{x}^{\tilde{\varepsilon}, \tilde{\rho}}$ and $\omega_{x}=\Omega_{x}^{\tilde{\varepsilon} / 2, \tilde{\rho} / 2}$. If $x \in \Omega$, by a similar argument we find a ball $B_{\tilde{\varepsilon}}(x)$ with $\tilde{\varepsilon} \in(0, \partial \Omega / 2)$ such that it holds that $\|u\|_{W^{1, p}\left(\partial B_{\tilde{\varepsilon}}(x)\right)} \lesssim c(d(x, \partial \Omega))\|u\|_{W^{1, p}\left(B_{\tilde{\varepsilon}}(x)\right)}$ and set $\Omega_{x}=B_{\tilde{\varepsilon}}(x), \omega_{x}=B_{\tilde{\varepsilon} / 2}(x)$. A standard compactness argument shows that the cover $\left\{\omega_{x}\right\}_{x \in \Omega}$ of $\Omega$ contains a finite subcover with the desired properties.

The next lemma enables us to to stretch a small neighbourhood of the boundary in a controlled manner. This will be crucial in constructing sequences with improved integrability but unchanged boundary behaviour.

Lemma 6 Suppose $\Omega$ is a $C^{1, \alpha}$-domain. Then there is a family of domains $\Omega^{s} \ni \Omega$ and a family of $C^{1, \alpha}$-diffeomorphisms $\Psi_{s}: \Omega^{s} \rightarrow \Omega$ such that

(i) $J \Psi_{s} \rightarrow 1$ and $\left|D \Psi_{s}-\mathrm{Id}\right| \rightarrow 0$ uniformly in $\Omega^{s}$ as $s \nearrow 1$. Equivalently, $J \Psi_{s}^{-1} \rightarrow 1$ and $\left|D \Psi_{s}^{-1}-\mathrm{Id}\right| \rightarrow 0$ uniformly in $\Omega$ as $s \nearrow 1$.

(ii) If $g \in W^{1+\frac{1}{q}, q}(\Omega)$ there is an extension $\hat{g}$ of $g$ to $\Omega^{s}$ such that $\hat{g} \in W^{1, q}\left(\Omega^{s}\right)$ and $\hat{g} \circ \Psi_{s}^{-1} \in g+W^{1, q}(\Omega)$.

Proof Let $X \in C^{\infty}\left(\mathbb{R}^{n}, \mathbb{R}^{n}\right)$ be a smooth vector field transversal to $\partial \Omega$. Fix $t_{0}>0$. Given $z \in \partial \Omega$ and for $|t| \leq 2 t_{0}$ consider the flow

$$
\begin{aligned}
\frac{d h_{z}}{d t} & =X(h(t)) \\
h_{z}(s) & =z
\end{aligned}
$$

and set $\Psi(z, t)=h_{z}(t)$. After possibly reducing the value of $t_{0}$, the maps $\Psi, \Psi^{-1}$ are $C^{1, \alpha}$-regular diffeomorphisms on a neighbourhood of $\partial \Omega$ which we denote $V$. Moreover the Jacobians of $\Psi, \Psi^{-1}$ are bounded.

Let $\frac{1}{2} \leq s<1$. Consider $\tau_{s}:\left[-t_{0}, t_{0}\right] \rightarrow\left[-t_{0}, t_{0}\right]$, a sequence of strictly monotonically increasing smooth maps with

$$
\tau_{s}\left(-t_{0}\right)=-t \quad \tau_{s}\left(t_{0}\right)=s^{-1} t_{0} \quad \tau_{s}^{\prime}\left(-t_{0}\right)=-1
$$


and such that $\tau_{s} \rightarrow \operatorname{Id}$ in $C^{2}\left(\left[-t_{0}, t_{0}\right]\right)$ as $s \rightarrow 1$. Define

$$
\Psi_{s}^{-1}(x)= \begin{cases}\Psi\left(x_{0}, \tau_{s}(t)\right) & \text { for } x=\Psi\left(x_{0}, t\right) \in V \\ x & \text { else } .\end{cases}
$$

Set $\Omega^{s}=\Psi_{s}^{-1}(\Omega) \subset V \cup \Omega$. Using the chain rule we note that $\Psi_{s}^{-1}: \Omega \rightarrow \Omega^{s}$ is a $C^{1, \alpha}$-regular diffeomorphism. Denote its inverse by $\Psi_{s}: \Omega^{s} \rightarrow \Omega$ and note that using the Inverse Function Theorem and the chain rule $\Psi_{s} \rightarrow \operatorname{Id}$ in $C^{1}\left(\Omega^{s}\right)$ as $s \nearrow 1$. In particular, also $J \Psi_{s} \rightarrow 1$ uniformly in $\Omega^{s}$ as $s \nearrow 1$. To obtain $\hat{g}$, simply set

$$
\hat{g}(x)= \begin{cases}g\left(x_{0}, 0\right) & \text { for } x=\Psi\left(x_{0}, t_{0}\right) \in \Omega^{s} \backslash \Omega \\ g(x) & \text { for } x \in \Omega .\end{cases}
$$

We conclude this section by noting a number of extensions we may carry out if $\Omega$ is a Lipschitz domain: Let $\Omega \Subset B(0, R)$. Due to [42], if $g \in W^{s, p}(\Omega)$, then there is an extension $\tilde{g} \in W^{s, p}\left(\mathbb{R}^{n}, \mathbb{R}^{m}\right)$ with

$$
\|\tilde{g}\|_{W^{s, p}\left(\mathbb{R}^{n}\right)} \lesssim\|g\|_{W^{s, p}(\Omega)} .
$$

Further we extend $F(x, z)$ to a functional on $B(0, R) \times \mathbb{R}^{n \times m}$, still denoted $F(x, z)$, that satisfies

$$
\begin{aligned}
|F(x, z)-F(y, z)| & \lesssim \Lambda|x-y|^{\alpha}\left(1+|z|^{2}\right)^{\frac{q}{2}} \\
\mid & F(x, z) \mid \lesssim \Lambda\left(1+|z|^{2}\right)^{\frac{q}{2}}
\end{aligned}
$$

by setting for $x \in B(0, R) \backslash \Omega$,

$$
F(x, z)=\inf _{y \in \Omega}\left(F(y, z)+\Lambda\left(1+|z|^{2}\right)^{\frac{q}{2}}|x-y|\right) .
$$

\subsection{Whitney-Besicovitch coverings}

In this section we define Whitney-Besicovitch coverings which will be a key tool in our proof of regularity for pointwise minimisers. Whitney-Besicovitch coverings combine properties of Whitney and Besicovitch coverings and were introduced in [33]. A nice presentation of the theory is given in [34]. To be precise:

Definition 2 A family of dyadic cubes $\left\{Q_{i}\right\}_{i \in I}$ with mutually disjoint interiors is called a Whitney-covering of $\Omega$ if

$$
\begin{gathered}
\bigcup_{i \in I} Q_{i}=\bigcup_{i \in I} 2 Q_{i}=\Omega \\
5 Q_{i} \cap\left(\mathbb{R}^{n} \backslash \Omega\right) \neq \emptyset .
\end{gathered}
$$

A family of cubes $\left\{K_{i}\right\}_{i \in I}$ is called a Whitney-Besicovitch-covering (WB-covering) of $\Omega$ if there is a triple $(\delta, M, \varepsilon)$ of positive numbers such that

$$
\begin{aligned}
& \bigcup_{i \in I} \frac{1}{1+\delta} K_{i}=\bigcup_{i \in I} K_{i}=\Omega \\
& \sum_{i \in I} \chi_{K_{i}} \leq M
\end{aligned}
$$




$$
K_{i} \cap K_{j} \neq \emptyset \Rightarrow\left|K_{i} \cap K_{j}\right| \geq \varepsilon \max \left(\left|K_{i}\right|,\left|K_{j}\right|\right) .
$$

The existence of a Whitney-covering for $\Omega$ is classical. The refinement to a WB-covering can be found in [34]:

Theorem 4 (cf. Theorem 3.15, [34]) Let $\Omega$ be an open subset of $\mathbb{R}^{n}$ with non-empty complement. Let $\left\{Q_{i}\right\}$ be a family of cubes which are a Whitney covering of $\Omega$. Then the cubes $K_{i}=\left(1+\frac{1}{6}\right) Q_{i}$ are a WB-covering of $\Omega$ with $\delta=\frac{1}{6}, \varepsilon=\frac{1}{14^{n}}$ and $M \leq 6^{n}-4^{n}+1$. Moreover for this covering $\frac{2}{\left(1+\frac{1}{6}\right)^{\frac{1}{n}}}\left|K_{i}\right|^{\frac{1}{n}} \leq \operatorname{dist}\left(K_{i}, \partial \Omega\right)$.

It will be of crucial importance to us that there exists a partition of unity associated to a WB-covering.

Theorem 5 (cf. Theorem 3.19, [34]) Suppose the cubes $\left\{K_{i}\right\}_{i \in I}$ form a WB-covering of $\Omega$ with constants $(\delta, M, \varepsilon)$. Then there is a family $\left\{\psi_{i}\right\}_{i \in I}$ of infinitely differentiable functions that form a partition of unity on $\Omega$ with the following properties:

$$
\begin{aligned}
& \operatorname{supp}\left(\psi_{i}\right) \subset \frac{1+\frac{\delta}{2}}{1+\delta} K_{i} \\
& \psi_{i}(x) \geq \frac{1}{M} \text { for } x \in \frac{1}{1+\delta} K_{i} \\
& \left|D \psi_{i}\right| \leq c \frac{1}{\left|K_{i}\right|^{\frac{1}{n}}} .
\end{aligned}
$$

for all $i \in I$.

\subsection{Properties of the relaxed and the regularised functional}

In this section, we collect some results regarding the relaxed function $\overline{\mathscr{F}}(\cdot)$ and its relation to a regularised version of $\mathscr{F}(\cdot)$. For local versions of $\overline{\mathscr{F}}(\cdot)$ these results are well-known, c.f. Section 6 in [36] and [19]. Define for $u \in W^{1, p}(\Omega)$

$$
\mathscr{F}_{\varepsilon}(u)= \begin{cases}\mathscr{F}(u)+\varepsilon \int_{\Omega}|D u|^{q} \mathrm{~d} x & \text { if } u \in W^{1, q}(\Omega) \\ \infty & \text { else. }\end{cases}
$$

Write $F_{\varepsilon}(x, z)=F(x, z)+\varepsilon|z|^{q}$. If $F(x, z)$ is measurable in $x$ and continuously differentiable in $z$, it is easy to see that so is $F_{\varepsilon}(x, z)$. Moreover if $F(x, z)$ satisfies (H1)-(H3), then $F_{\varepsilon}(x, z)$ satisfies the bounds

$$
\begin{aligned}
& v\left(\mu^{2}+|z|^{2}+|w|^{2}\right)^{\frac{p-2}{2}} \leq \frac{F_{\varepsilon}(x, z)-F_{\varepsilon}(x, w)-\partial_{z} F_{\varepsilon}(x, w) \cdot(z-w)}{|z-w|^{2}} \\
& \left|F_{\varepsilon}(x, z)\right| \leq(\Lambda+\varepsilon)\left(1+|z|^{2}\right)^{\frac{q}{2}} \\
& \left|\partial_{z} F_{\varepsilon}(x, z)\right| \lesssim(\Lambda+\varepsilon)\left(1+|z|^{2}\right)^{\frac{q-1}{2}} \\
& \left|F_{\varepsilon}(x, z)-F_{\varepsilon}(y, z)\right| \leq \Lambda|x-y|^{\alpha}\left(1^{2}+|z|^{2}\right)^{\frac{q}{2}} .
\end{aligned}
$$

Further note that if $F(x, z)$ satisfies (H4), then so does $F_{\varepsilon}(x, z)$.

Minimisers of $\mathscr{F}_{\varepsilon}(\cdot)$ and $\overline{\mathscr{F}}(\cdot)$ are related as follows:

Lemma 7 (c.f. Lemma 6.4. in [36]) Let $g \in W^{1, q}(\Omega)$. Suppose $F(x, z)$ satisfies (H1) and $(\mathrm{H} 2)$. Suppose $u$ is a relaxed minimiser of $\mathscr{F}(\cdot)$ in the class $W_{g}^{1, p}(\Omega)$ and $u_{\varepsilon}$ is the pointwise 
minimiser of $\mathscr{F}_{\varepsilon}(\cdot)$ in the class $W_{g}^{1, q}(\Omega)$. Then $\mathscr{F}_{\varepsilon}\left(u_{\varepsilon}\right) \rightarrow \overline{\mathscr{F}}(u)$ as $\varepsilon \rightarrow 0$. Moreover up to passing to a subsequence $u_{\varepsilon} \rightarrow u$ in $W^{1, p}(\Omega)$.

Proof Note that by our assumptions $f \cdot u \in L^{1}(\Omega)$. Hence existence and uniqueness of $u_{\varepsilon}$ follows from the direct method and strict convexity, respectively. We further note that

$$
\overline{\mathscr{F}}(u) \leq \liminf _{\varepsilon \rightarrow 0} \mathscr{F}\left(u_{\varepsilon}\right) \leq \liminf _{\varepsilon \rightarrow 0} \mathscr{F}\left(u_{\varepsilon}\right) .
$$

To prove the reverse implication note that for any $v \in W_{g}^{1, q}(\Omega)$,

$$
\limsup _{\varepsilon \rightarrow 0} \mathscr{F}_{\varepsilon}\left(u_{\varepsilon}\right) \leq \lim _{\varepsilon \rightarrow 0} \mathscr{F}_{\varepsilon}(v)=\mathscr{F}(v)=\overline{\mathscr{F}}(v) .
$$

By definition of $\overline{\mathscr{F}}(\cdot)$ the inequality above extends to all $v \in W_{g}^{1, p}(\Omega)$. In particular, it holds with the choice $v=u$. Thus $\mathscr{F}_{\varepsilon}\left(u_{\varepsilon}\right) \rightarrow \overline{\mathscr{F}}(u)$.

Using (H1) we may extract a (non-relabelled) subsequence of $u_{\varepsilon}$ so that $u_{\varepsilon} \rightarrow v$ weakly in $W^{1, p}(\Omega)$ for some $v \in W^{1, p}(\Omega)$. Note using our calculations above that $v$ is a relaxed minimiser of $\mathscr{F}(\cdot)$ in the class $W_{g}^{1, p}(\Omega)$. Using $(\mathrm{H} 1)$ it is easy to see that for $w_{1}, w_{2} \in$ $W_{g}^{1, q}(\Omega)$,

$$
\overline{\mathscr{F}}\left(\frac{w_{1}+w_{2}}{2}\right)+\frac{v}{p}\left\|D w_{1}-D w_{2}\right\|_{L^{p}(\Omega)}^{p} \leq \frac{1}{2}\left(\overline{\mathscr{F}}\left(w_{1}\right)+\overline{\mathscr{F}}\left(w_{2}\right)\right) .
$$

Using the definition of $\overline{\mathscr{F}}(\cdot)$ and weak lower semicontinuity of norms, we see that this estimate extends to $w_{1}, w_{2} \in W_{g}^{1, p}(\Omega)$. In particular, $\overline{\mathscr{F}}(\cdot)$ is convex and so $u=v$. Moreover the choice $w_{1}=u, w_{2}=u_{\varepsilon}$ in the estimate shows that $u_{\varepsilon} \rightarrow u$ in $W^{1, p}(\Omega)$.

A useful criterion for establishing the equality $\overline{\mathscr{F}}(u)=\mathscr{F}(u)$ is the following:

Lemma 8 (cf. [8]) Let $1<p \leq q<\infty$. For $u \in W_{g}^{1, p}(\Omega)$ with $\mathscr{F}(u)<\infty$, we have $\overline{\mathscr{F}}(u)=\mathscr{F}(u)$ if and only if there is a sequence $u_{k} \in W_{g}^{1, q}(\Omega)$ such that $u_{k} \rightarrow u$ weakly in $W^{1, p}(\Omega)$ and $\mathscr{F}\left(u_{\varepsilon}\right) \rightarrow \mathscr{F}(u)$ as $\varepsilon \rightarrow 0$.

We close this section by showing that relaxed minimisers are very weak solutions of the Euler-Lagrange system. We first recall a Lemma from [15].

Lemma 9 Suppose $F(x, z)$ satisfies $(\mathrm{H} 1)$ and $(\mathrm{H} 2)$. Then

$$
\partial_{z} F(z) \cdot z \gtrsim|z|^{q}+\left|\partial_{z} F(z)\right|^{q^{\prime}}-1 .
$$

Proof Consider the Fenchel-conjugate of the partial integrand $F(x, z)$,

$$
F^{*}(x, \xi)=\sup _{\zeta \in \mathbb{R}^{n \times m}}(\xi \cdot \zeta-F(x, \zeta)) \quad(x, \xi) \in \Omega \times \mathbb{R}^{n \times m} .
$$

The Fenchel-Young inequality in the extremal case yields that

$$
\partial_{z} F(z) \cdot z=F(x, z)+F^{*}\left(z, \partial_{z} F(x, z)\right) .
$$

To conclude note by direct calculation $F^{*}\left(x, \partial_{z} F(x, z)\right) \gtrsim\left|\partial_{z} F(x, \xi)\right|^{q^{\prime}}-1$.

We adapt an argument from [15]. 
Lemma 10 Let $g \in W^{1, q}(\Omega)$ and suppose $F(x, z)$ satisfies $(\mathrm{H} 1)$ and $(\mathrm{H} 2)$. Let $u$ be a relaxed minimiser of $\mathscr{F}(\cdot)$ in the class $W_{g}^{1, p}(\Omega)$. Then we have $\partial_{z} F(x, D u) \in L^{q^{\prime}}(\Omega)$ and

$$
\int_{\Omega} \partial_{z} F(x, D u) \cdot D \psi-f \psi \mathrm{d} x=0 \quad \forall \psi \in W_{0}^{1, q}(\Omega) .
$$

Proof By the direct method we obtain $u_{\varepsilon} \in W_{g}^{1, q}(\Omega)$ minimising $\mathscr{F}_{\varepsilon}(\cdot)$ in the class $W_{g}^{1, q}(\Omega)$. Denote $\sigma_{\varepsilon}=\partial_{z} F_{\varepsilon}\left(\cdot, D u_{\varepsilon}\right)$ and $\mu_{\varepsilon}=\left|D u_{\varepsilon}\right|^{q-2} D u_{\varepsilon}$. Then $u_{\varepsilon}$ satisfies the Euler-Lagrange system

$$
\int_{\Omega}\left(\sigma_{\varepsilon}+\varepsilon \mu_{\varepsilon}\right) \cdot D \psi-f \psi \mathrm{d} x=0 \quad \forall \psi \in W_{0}^{1, q}(\Omega) .
$$

Choose $\psi=u_{\varepsilon}-g$ and use Lemma 9, Hölder and Young's inequality to find that for any $\delta>0$,

$$
\begin{aligned}
\int_{\Omega}\left|D u_{\varepsilon}\right|^{p}+\left|\sigma_{\varepsilon}\right|^{q^{\prime}}+\varepsilon\left|D u_{\varepsilon}\right|^{q} \mathrm{~d} x & \lesssim C \int_{\Omega}\left(\sigma_{\varepsilon}+\varepsilon \mu_{\varepsilon}\right) \cdot D u_{\varepsilon} \mathrm{d} x \\
= & C \int_{\Omega}\left(\sigma_{\varepsilon}+\varepsilon \mu_{\varepsilon}\right) \cdot D g+f \cdot\left(u_{\varepsilon}-g\right) \mathrm{d} x \\
\leq & \delta\left(\left\|\sigma_{\varepsilon}\right\|_{L^{q^{\prime}(\Omega)}}^{q^{\prime}}+\left\|u_{\varepsilon}\right\|_{L^{q}(\Omega)}^{q}+\varepsilon\left\|D u_{\varepsilon}\right\|_{L^{q}(\Omega)}^{q}\right) \\
& +C(\delta)\left(1+\|g\|_{W^{1, q}(\Omega)}^{q}+\|f\|_{L^{q^{\prime}(\Omega)}}^{q^{\prime}}\right)
\end{aligned}
$$

Noting that $\left\|u_{\varepsilon}\right\|_{L^{q}(\Omega)} \lesssim\left\|D u_{\varepsilon}\right\|_{L^{p}(\Omega)}$ by our choice of $q$, after choosing $\delta$ sufficiently small we may rearrange to conclude

$$
\limsup _{\varepsilon \searrow 0} \int_{\Omega}\left|\sigma_{\varepsilon}\right|^{q^{\prime}} \mathrm{d} x \lesssim 1+\int_{\Omega}|f|^{q^{\prime}}+|g|^{q}+|D g|^{q} \mathrm{~d} x .
$$

Since $\partial_{z} F(x, z)$ is Carathéodory and $D u_{\varepsilon} \rightarrow D u$ in measure by Lemma 7, we also have $\sigma_{\varepsilon} \rightarrow F_{z}(\cdot, D u)$ in measure. Finally note that $\sigma_{\varepsilon}+\varepsilon \mu_{\varepsilon} \rightarrow F_{z}(\cdot, D u)$ in $L^{1}(\Omega)$, so the latter is row-wise divergence free and since it is also in $L^{q^{\prime}}(\Omega)$, the result is established.

\section{Regularity of minimisers of the relaxed functional}

The aim of this section is to prove Theorem 1. For the convenience of the reader we recall the statement:

Theorem 6 Suppose $F(x, \cdot)$ satisfies $(\mathrm{H} 1)-(\mathrm{H} 3)$ with $1<p \leq q<\frac{(n+\alpha) p}{n}$. Then if $u$ is a relaxed minimiser of $\mathscr{F}(\cdot)$ in the class $W_{g}^{1, p}(\Omega), u \in W^{1, q}(\Omega)$. Moreover for any $0 \leq \beta<\alpha$ there is $\gamma>0$ such that, we have the estimate

$$
\|u\|_{W^{1, \frac{n p}{n-\beta}(\Omega)}} \lesssim\left(1+\overline{\mathscr{F}}(u)+\|g\|_{W^{1+\alpha, q}(\Omega)}+\|f\|_{L^{q^{\prime}(\Omega)}}\right)^{\gamma} .
$$

The key to proving the theorem is an a-priori estimate for minimisers of the regularised functional:

Lemma 11 Suppose the assumptions of Theorem 1 hold. Let $v_{\varepsilon}$ be the minimiser of $\mathscr{F}_{\varepsilon}(\cdot)$ in the class $W_{g}^{1, q}(\Omega)$. Then for any $0 \leq \beta<\alpha$ there is $\gamma>0$ such that the estimate

$$
\left\|v_{\varepsilon}\right\|_{W^{1, \frac{n p}{n-\beta}(\Omega)}} \lesssim\left(1+\mathscr{F}\left(v_{\varepsilon}\right)+\|g\|_{W^{1+\alpha, q}(\Omega)}+\|f\|_{L^{q^{\prime}}(\Omega)}\right)^{\gamma}
$$


holds, with the implicit constant independent of $\varepsilon$ and $\gamma$.

Theorem 1 is now a consequence of combining Lemmas 11 and 7.

Proof of Theorem 1 Let $v_{\varepsilon} \in W_{g}^{1, q}(\Omega)$ be the minimiser of $\mathscr{F}_{\varepsilon}(\cdot)$ in the class $W_{g}^{1, q}(\Omega)$. For $0 \leq \beta<\alpha$ by Lemma 11, $\left\{v_{\varepsilon}\right\}$ is uniformly bounded in $W^{1, \frac{n p}{n-\beta}}(\Omega)$. From Lemma 7 we know that $\mathscr{F}_{\varepsilon}\left(v_{\varepsilon}\right) \rightarrow \overline{\mathscr{F}}(u)$ and up to passing to a subsequence $v_{\varepsilon} \rightarrow u$ in $W^{1, p}(\Omega)$. Thus we may extract a non-relabelled subsequence such that $v_{\varepsilon} \rightarrow u$ weakly in $W^{1, \frac{n p}{n-\beta}}(\Omega)$. This allows to pass to the limit in Lemma 11 to conclude.

We proceed to prove Lemma 11:

Proof of Lemma 11 By direct methods and strict convexity deriving from (12) we obtain a sequence of minimisers $v_{\varepsilon} \in W_{0}^{1, q}(\Omega)$ that solve

$$
\min _{v \in W_{0}^{1, q}(\Omega)} \mathscr{F}_{\varepsilon}(v+g)
$$

Moreover $v_{\varepsilon}$ satisfies the Euler-Lagrange equation

$$
\int_{\Omega}\left(\partial_{z} F_{\varepsilon}\left(x, D v_{\varepsilon}+D g\right)\right) \cdot D \phi-f \phi \mathrm{d} x=0 \quad \forall \phi \in W_{0}^{1, q}(\Omega) .
$$

In particular, for any $v \in W_{0}^{1, q}(\Omega)$,

$$
\int_{\Omega}\left(\partial_{z} F_{\varepsilon}\left(x, D v_{\varepsilon}+D g\right)\right) \cdot D\left(v-v_{\varepsilon}\right)-f \cdot\left(v-v_{\varepsilon}\right)=0 .
$$

Using this identity, we see for $v \in W_{0}^{1, q}(\Omega)$,

$$
\begin{aligned}
& \mathscr{F}_{\varepsilon}(v+g)-\mathscr{F}_{\varepsilon}\left(v_{\varepsilon}+g\right) \\
&= \int_{\Omega} F_{\varepsilon}(x, D v+D g)-F_{\varepsilon}\left(x, D v_{\varepsilon}+D g\right)-f \cdot\left(v-v_{\varepsilon}\right) \mathrm{d} x \\
&-\int_{\Omega}\left(\partial_{z} F_{\varepsilon}\left(x, D v_{\varepsilon}+D g\right)\right) \cdot D\left(v-v_{\varepsilon}\right)-f \cdot\left(v-v_{\varepsilon}\right) \mathrm{d} x \\
& \gtrsim\left\|V_{\mu, p}(D v+D g)-V_{\mu, p}\left(D v_{\varepsilon}+D g\right)\right\|_{L^{2}(\Omega)}^{2},
\end{aligned}
$$

where to obtain the last line we have used (12) and Lemma 1.

Let $\rho_{0}>0$ and $\boldsymbol{n}: \mathbb{R}^{n} \rightarrow S^{n-1}$ be so that the uniform cone property (7) holds. Possibly reducing $\rho_{0}$ assume without loss of generality that we have $\Omega+B_{3 \rho_{0}}(x) \subset B(0, R)$ for all $x \in \Omega$. Here $B(0, R) \ni \Omega$ is the ball defined in Sect. 2.3. Given $x_{0} \in \Omega$, let $0 \leq$ $\phi=\phi_{x_{0}, \rho_{0}} \leq 1$ be a smooth cut-off supported in $B_{2 \rho_{0}}\left(x_{0}\right)$ with $\phi(x)=1$ in $B_{\rho_{0}}\left(x_{0}\right)$ and $\left|D^{k} \phi(x)\right| \leq C_{k} \rho_{0}{ }^{-k}$ for some $C_{k}>0$ and $k \in N$. Given functions $v_{1}, v_{2}$ defined on $\mathbb{R}^{n}$ and $h \in \mathbb{R}^{n}$ introduce

$$
T_{h} v_{1}=\phi v_{1, h}+(1-\phi) v_{1}
$$

and denote $\Delta_{h} v_{1}=v_{1, h}-v_{1}$. If $v_{1}, v_{2}: \mathbb{R}^{n} \rightarrow \mathbb{R}^{n \times m}$ then write

$$
\tilde{\Delta}_{h} F_{\varepsilon}\left(x, v_{1}, v_{2}\right)=F_{\varepsilon}\left(x, v_{1, h}+v_{2}\right)-F_{\varepsilon}\left(x, v_{1}+v_{2}\right) .
$$

We claim that for every $x_{0} \in \mathbb{R}^{n}$ there is a constant $C=C\left(n, \rho_{0}, \Lambda, \Omega\right)$ such that for all $v \in W_{0}^{1, q}(\Omega)$

$$
\sup _{h \in C_{\rho_{0}}\left(\theta_{0}, \boldsymbol{n}\left(x_{0}\right)\right)} \frac{\mathscr{F}_{\varepsilon}\left(T_{h} \tilde{v}+g\right)-\mathscr{F}_{\varepsilon}(v+g)}{|h|^{\alpha}}
$$




$$
\leq C\left(1+\|D v\|_{L^{q}(\Omega)}^{q}+\|g\|_{W^{1+\alpha, q}(\Omega)}^{q}+\|f\|_{L^{q^{\prime}(\Omega)}}^{q^{\prime}}\right)
$$

Here $\tilde{v}$ is the extension by 0 to $\mathbb{R}^{n}$ of $v$.

Let $v \in W_{0}^{1, q}(\Omega)$ and $h \in C_{\rho_{0}}\left(\theta_{0}, \boldsymbol{n}\left(x_{0}\right)\right)$. Let $\tilde{v}$ be the extension of $v$ by 0 to a function on $\mathbb{R}^{n}$. We estimate for $h \in C_{\rho_{0}}\left(\theta_{0}, \boldsymbol{n}\left(x_{0}\right)\right)$,

$$
\begin{aligned}
\mathscr{F}_{\varepsilon} & \left(T_{h} \tilde{v}+g\right)-\mathscr{F}_{\varepsilon}(v+g) \\
= & \int_{\Omega} F_{\varepsilon}\left(x, T_{h} D \tilde{v}+D \phi\left(\tilde{v}_{h}-v\right)+D g\right)-F_{\varepsilon}\left(x, T_{h} D \tilde{v}+D g\right) \mathrm{d} x \\
& +\int_{\Omega} F_{\varepsilon}\left(x, T_{h} D \tilde{v}+D g\right)-F_{\varepsilon}(x, D \tilde{v}+D g) \mathrm{d} x-\int_{\Omega} f \cdot\left(T_{h} \tilde{v}-v\right) \mathrm{d} x \\
= & A_{1}+A_{2}+A_{3} .
\end{aligned}
$$

Using (13), Hölder's inequality and recalling that $\tilde{g}$ denotes a $W^{1+\alpha, q}$-extension of $g$ to $\mathbb{R}^{n}$, we find

$$
\begin{aligned}
\left|A_{1}\right| \leq & (\Lambda+\varepsilon) \int_{\Omega}\left|D \phi \Delta_{h} \tilde{v}\right|\left(1+\left|T_{h} D \tilde{v}+D g\right|^{2}+\left|D \phi \Delta_{h} \tilde{v}\right|^{2}\right)^{\frac{q-1}{2}} \mathrm{~d} x \\
\leq & \left\|D \phi \Delta_{h} \tilde{v}\right\|_{L^{q}\left(B_{2 \rho_{0}}\left(x_{0}\right)\right)}\left(1+\left\|T_{h} D \tilde{v}\right\|_{L^{q}\left(B_{2 \rho_{0}}\left(x_{0}\right)\right)}\right. \\
& \left.+\left\|D \phi \Delta_{h} \tilde{v}\right\|_{L^{q}\left(B_{2 \rho_{0}}\left(x_{0}\right)\right)}+\|D \tilde{g}\|_{L^{q}\left(B_{2 \rho_{0}}\left(x_{0}\right)\right)}^{q}\right)^{q-1} .
\end{aligned}
$$

Recalling that as $\tilde{v} \in W_{0}^{1, q}\left(\Omega, \mathbb{R}^{m}\right)$,

$$
\left\|D \phi\left(\tilde{v}_{h}-\tilde{v}\right)\right\|_{L^{q}\left(B_{2 \rho_{0}}\left(x_{0}\right)\right)} \lesssim|h|\|D \tilde{v}\|_{L^{q}\left(B_{2 \rho_{0}}\left(x_{0}\right)\right)},
$$

we conclude using (8) and the definition of $T_{h}$,

$$
\left|A_{1}\right| \lesssim|h|\left(1+\|D v\|_{L^{q}(\Omega)}^{q}+\|g\|_{W^{1+\alpha, q}(\Omega)}^{q}\right) .
$$

We now turn to $A_{2}$. By convexity of $F_{\varepsilon}(\cdot)$,

$$
\begin{aligned}
\tilde{\Delta}_{h} F_{\varepsilon}(x, D \tilde{v}) & \leq(1-\phi) F_{\varepsilon}(x, D v+D g)+\phi F_{\varepsilon}\left(x, D \tilde{v}_{h}+D g\right)-F_{\varepsilon}(D v+D g) \\
& =\phi \tilde{\Delta}_{h} F_{\varepsilon}(x, D \tilde{v}, D \tilde{g}) .
\end{aligned}
$$

In particular, using a change of coordinates and since in $B_{3 \rho} \backslash \Omega, D \tilde{v}_{h}=D \tilde{v}=0$,

$$
\begin{aligned}
\left|A_{2}\right| \leq & \int_{B_{2 \rho_{0}}\left(x_{0}\right)} \phi \tilde{\Delta}_{h} F_{\varepsilon}(x, D \tilde{v}+D g) \mathrm{d} x \\
= & \int_{B_{2 \rho_{0}}\left(x_{0}\right)+h} \phi(x-h) F_{\varepsilon}\left(x-h, D \tilde{v}+D \tilde{g}_{-h}\right) \mathrm{d} x \\
& -\int_{B_{2 \rho_{0}}\left(x_{0}\right)} \phi(x) F_{\varepsilon}(x, D \tilde{v}+D \tilde{g}) \mathrm{d} x \\
= & \int_{B_{3 \rho_{0}}\left(x_{0}\right)} \Delta_{-h} \phi F_{\varepsilon}(x-h, D \tilde{v}+D \tilde{g}-h)+\phi(x) \tilde{\Delta}_{-h} F_{\varepsilon}(x-h, D \tilde{g}+D \tilde{v}) \mathrm{d} x \\
& +\int_{B_{2 \rho_{0}}\left(x_{0}\right)} \phi(x)\left(F_{\varepsilon}(x-h, D \tilde{v}+D \tilde{g})-F_{\varepsilon}(x, D \tilde{v}+D \tilde{g})\right) \mathrm{d} x .
\end{aligned}
$$

Using (13), (15), the regularity of $\phi$ and $g$ as well as (8) to estimate each term in turn, we conclude

$$
A_{2} \lesssim|h|^{\alpha}\left(1+\|D v\|_{L^{q}(\Omega)}^{q}+\|g\|_{W^{1+\alpha, q}(\Omega)}^{q}\right) .
$$


Finally, using Young's inequality and Sobolev embedding,

$$
\left|A_{3}\right| \leq \int_{\Omega}\left|f \cdot\left(\phi \Delta_{h} v\right)\right| \lesssim\|f\|_{L^{q^{\prime}(\Omega)}}\left\|v-v_{h}\right\|_{L^{q}(\Omega)} \lesssim h\left(\|f\|_{L^{q^{\prime}(\Omega)}}^{q^{\prime}}+\|D v\|_{L^{q}(\Omega)}^{q}\right)
$$

This proves the claim.

We are now ready to finish the proof. Note that $T_{h} \tilde{v}_{\varepsilon} \in W_{0}^{1, q}(\Omega)$ if we choose $h \in C_{\rho_{0}}\left(\theta_{0}, \boldsymbol{n}\left(x_{0}\right)\right)$. Thus combining (17) and (18) and making the choice $v=T_{h} \tilde{v}_{\varepsilon}$ we find for $x_{0} \in \mathbb{R}^{n}$,

$$
\begin{aligned}
& \sup _{h \in C_{\rho_{0}}\left(\theta_{0}, \boldsymbol{n}\left(x_{0}\right)\right)}|h|^{-\alpha}\left\|V_{\mu, p}\left(D v_{\varepsilon}+D g\right)-V_{\mu, p}\left(D T_{h} \tilde{v}_{\varepsilon, h}+D g\right)\right\|_{L^{2}\left(B_{\rho_{0}}\left(x_{0}\right)\right)}^{2} \\
& \quad \lesssim 1+\|D w\|_{L^{q}(\Omega)}^{q}+\|g\|_{W^{1+\alpha, q}(\Omega)}^{q}+\|f\|_{L^{q^{\prime}(\Omega)}}^{q^{\prime}} .
\end{aligned}
$$

In particular, we deduce after recalling the definition of $T_{h}$, using the triangle inequality and regularity of $\tilde{g}$,

$$
\begin{aligned}
& \sup _{h \in C_{\rho_{0}}\left(\theta_{0}, \boldsymbol{n}\left(x_{0}\right)\right)}|h|^{-\alpha}\left\|D\left(\tilde{v}_{\varepsilon, h}+\tilde{g}_{h}\right)-D\left(v_{\varepsilon}+g\right)\right\|_{L^{p}\left(B_{\rho_{0}}\left(x_{0}\right)\right.}^{p} \\
& \lesssim 1+\|D w\|_{L^{q}(\Omega)}^{q}+\|g\|_{W^{1+\alpha, q}(\Omega)}^{q}+\|f\|_{L^{q^{\prime}(\Omega)}}^{q^{\prime}} .
\end{aligned}
$$

Using the characterisation of Besov spaces (5), we conclude for all $x_{0} \in \mathbb{R}^{n}$,

$$
\left[D v_{\varepsilon}\right]_{\frac{\alpha}{p}, p, B_{\rho_{0}}\left(x_{0}\right)}^{p} \lesssim\left(1+\left\|D v_{\varepsilon}\right\|_{L^{q}(\Omega)}^{q}+\|g\|_{W^{1+\alpha, q}(\Omega)}^{q}+\|f\|_{L^{q^{\prime}(\Omega)}}^{q^{\prime}}\right)
$$

Covering $\Omega$ by a finite number of balls of radius $\rho_{0}$ and using (6), we conclude

$$
\left\|v_{\varepsilon}\right\|_{B_{\infty}^{1+\frac{\alpha}{p}, p}(\Omega)}^{p} \lesssim\left(1+\left\|v_{\varepsilon}\right\|_{W^{1, q}(\Omega)}^{q}+\|g\|_{W^{1+\alpha, q}(\Omega)}^{q}+\|f\|_{L^{q^{\prime}(\Omega)}}^{q^{\prime}}\right) .
$$

Recall now that $B_{\infty}^{1+\frac{\alpha}{p}, p}(\Omega)$ embeds continuously into $W^{1, \frac{n p}{n-\beta}}(\Omega)$ for any $\beta<\alpha$ by Theorem 3. Choose $\beta$ such that $q<\frac{p(\beta+n)}{n}$ and use interpolation with $\theta=\frac{n p}{\beta}\left(\frac{1}{p}-\frac{1}{q}\right)$ to see

$$
\left\|v_{\varepsilon}\right\|_{W^{1, q}(\Omega)} \leq\left\|v_{\varepsilon}\right\|_{W^{1, p}(\Omega)}^{1-\theta}\left\|v_{\varepsilon}\right\|_{W^{1, \frac{n p}{n-\beta}}(\Omega)}^{\theta}
$$

As, $q<\frac{(n+\beta) p}{n}$, it follows that $q \theta<p$. Thus using (22) in (21), we find after using Young's inequality,

$$
\begin{aligned}
\left\|v_{\varepsilon}\right\|_{W}^{p} & \lesssim \\
& +\|g\|_{W^{1+\alpha, q}(\Omega)}^{q}+\frac{1}{2}\left\|v_{\varepsilon}\right\|_{W^{1, \frac{n p}{n-\beta}}(\Omega)}^{p}+\|f\|_{L^{q^{\prime}}(\Omega)}^{q^{\prime}}
\end{aligned}
$$

Re-arranging and recalling (1) we obtain the desired conclusion.

Assume now $p<2$. In this case (19) does not hold in this form any more. Instead arguing as before up to this point and using Hölder, we find

$$
\begin{aligned}
& \left\|D v_{\varepsilon}+D g-D \tilde{v}_{\varepsilon, h}-D_{h} \tilde{g}\right\|_{L^{p}(\Omega)}^{2}\left(\int_{\Omega}\left|D v_{\varepsilon}\right|^{p}+\left|D \tilde{v}_{\varepsilon, h}\right|^{p}+|D g|^{p}+\left|D \tilde{g}_{h}\right|^{p} \mathrm{~d} x\right)^{\frac{p-2}{p}} \\
& \quad \lesssim 1+\|D w\|_{L^{q}(\Omega)}^{q}+\|g\|_{W^{1+\alpha, q}(\Omega)}^{q}+\|f\|_{L^{q^{\prime}(\Omega)}}^{q^{\prime}}
\end{aligned}
$$


In particular, proceeding as before we obtain

$$
\begin{aligned}
\left\|v_{\varepsilon}\right\|_{B_{\infty}^{1+\frac{\alpha}{2}}(\Omega)}^{2} \lesssim & \left(1+\left\|v_{\varepsilon}\right\|_{W^{1, q}(\Omega)}^{q}+\|g\|_{W^{1+\alpha, q}(\Omega)}^{q}+\|f\|_{L^{q^{\prime}(\Omega)}}^{q^{\prime}}\right) \\
& \times\left(\left\|v_{\varepsilon}\right\|_{W^{1, p}(\Omega)}+\|g\|_{W^{1, p}(\Omega)}\right)^{\frac{2-p}{p}} .
\end{aligned}
$$

It is now straightforward to check that applying the interpolation argument from the case $p \geq 2$ gives the desired conclusion.

Remark 1 In the case of autonomous functionals $F(x, z)=F(z)$ the results of this section may be strengthened as follows. Using techniques from interior regularity arguments, the Besov-regularity in tangential directions may be improved when compared to the Besovregularity obtained in the argument above. Combining this improvement with an embedding of anisotropic Besov spaces into Lebesgue spaces, $\frac{n p}{n-\beta}$ may be replaced by a larger value in (22). This in turn will imply that larger values of $q$ are allowed in the argument. We intend to return to this observation in further work.

\section{Regularisation and pointwise minimisers}

Throughout this section we set $\Theta=1+n\left(\frac{1}{q}-\frac{1}{p}\right)$ if $p<n, \Theta=\frac{n}{q}$ if $p \geq n$.

The aim of this section is to prove Theorem 2 and Proposition 1. In order to do so we require a number of technical lemmas, which we collect in the following subsection.

\subsection{Technical lemmas}

We begin with an observation already noted in [18, Theorem 3.1]. For completeness we give the proof.

Lemma 12 Assume $1<p \leq q \leq \frac{(n+\alpha) p}{n}$. Let $\Omega$ be a domain and $u \in W^{1, p}(\Omega)$. Suppose $F(x, \cdot)$ satisfies $(\mathrm{H} 1)-(\mathrm{H} 3)$ and $(\mathrm{H} 4)$. Then for $x \in \Omega$ and iffurther $\varepsilon \leq \min \left(\varepsilon_{0}, d(x, \partial \Omega)\right)$,

$$
F\left(x, D u(\cdot) \star \phi_{\varepsilon}(x)\right) \lesssim 1+\left(F(\cdot, D u(\cdot)) \star \phi_{\varepsilon}\right)(x) .
$$

Proof Let $C>0$ be an arbitrary fixed constant. We claim that if $|z| \leq C \varepsilon^{-\frac{n}{p}}$ and $x \in$ $\overline{B_{\varepsilon}\left(x_{0}\right) \cap \Omega}$, then

$$
F(x, z) \lesssim 1+\min _{y \in \overline{B_{\varepsilon}(x) \cap \Omega}} F(y, z) .
$$

Set $G_{\varepsilon}(x, z)=\min _{y \in \overline{B_{\varepsilon}(x) \cap \Omega}} F(x, z)$. By (H3) we deduce

$$
G_{\varepsilon}(x, z) \geq F(x, z)-\Lambda \varepsilon^{\alpha}\left(1+|z|^{2}\right)^{\frac{q}{2}} .
$$

Hence for $\delta \in(0,1), \varepsilon \in\left(0, \varepsilon_{0}\right)$ and $|z| \leq C \varepsilon^{-\frac{n}{p}}$, using (1) to note that $G_{\varepsilon}(x, z) \gtrsim|z|^{p}-1$,

$$
\begin{aligned}
G_{\varepsilon}(x, z) & =\delta G_{\varepsilon}(x, z)+(1-\delta) G_{\varepsilon}(x, z) \\
& \gtrsim \delta F(x, z)-\delta \Lambda \varepsilon^{\alpha}\left(1+|z|^{2}\right)^{\frac{q}{2}}+(1-\delta)\left(|z|^{p}-1\right) \\
& \gtrsim \delta F(x, z)-\delta \Lambda \varepsilon^{\alpha}|z|^{q}+(1-\delta)|z|^{p}-\delta \Lambda \varepsilon^{\alpha}-1 \\
& \geq \delta F(x, z)-\delta \Lambda C^{q-p} \varepsilon^{\alpha+\frac{n(p-q)}{p}}|z|^{p}+(1-\delta)|z|^{p}-\delta \Lambda \varepsilon^{\alpha}-1
\end{aligned}
$$




$$
\geq \delta F(x, z)+\left(1-\delta-\Lambda C^{q-p} \delta \varepsilon_{0}^{\alpha+\frac{n(p-q)}{p}}\right)|z|^{p}-\Lambda \varepsilon_{0}^{\alpha}-1
$$

The last line relies on $q \leq \frac{(n+\alpha) p}{n}$. Choosing $\delta$ sufficiently small we achieve that $1-\delta-$ $\Lambda C^{q-p} \delta \varepsilon_{0}{ }^{\alpha+\frac{n(p-q)}{p}} \geq 0$ and the second term on the last line may be dropped. Re-arranging (23) then follows.

Note that by Lemma 2

$$
\left|D u \star \phi_{\varepsilon}\right| \leq\|D u\|_{L^{p}(\Omega)} \varepsilon^{-\frac{n}{p}} .
$$

Using Jensen's inequality we may estimate for $\varepsilon \in\left(0, \varepsilon_{0}\right)$ with some fixed $\hat{y} \in B_{\varepsilon}(x)$,

$$
\begin{aligned}
G_{\varepsilon}\left(x, D u \star \phi_{\varepsilon}\right) & =F\left(\hat{y}, D u \star \phi_{\varepsilon}\right) \leq \int_{B_{1}} F(\hat{y}, D u(y)) \phi_{\varepsilon}(x-y) \mathrm{d} y \\
& \leq\left(F(\cdot, D u(\cdot)) \star \phi_{\varepsilon}\right)(x)
\end{aligned}
$$

To obtain the second inequality (H4) was used. In particular, using (23) we conclude

$$
F\left(x, D u \star \phi_{\varepsilon}\right) \lesssim 1+\left(F(\cdot, D u(\cdot)) \star \phi_{\varepsilon}\right)(x) .
$$

Partitions of unity feature heavily in our approximation approach. The next lemma studies the behaviour of $F(x, z)$ when $z$ is a sum built using a partition of unity.

Lemma 13 Let $\Omega$ be a domain with $\Omega=\bigcup_{i \in I} \Omega_{i}$, where $\Omega_{i} \subset \Omega$ is open relative to $\Omega$. Suppose $1<p \leq q<\frac{(n+1) p}{n}$ and suppose $\left\{\psi_{i}\right\}_{i \in I}$ is a partition of unity subordinate to $\left\{\Omega_{i}\right\}$ and such that

$$
\left\|D \psi_{i}\right\|_{L^{\infty}\left(\Omega_{i}\right)} \sim\left\|D \psi_{j}\right\|_{L^{\infty}\left(\Omega_{j}\right)} \quad \text { if } \operatorname{supp} \psi_{i} \cap \operatorname{supp} \psi_{j} \neq \emptyset .
$$

Assume further that there is $M>0$ such that for every $x \in \Omega$,

$$
\#\left\{i \in I: x \in \operatorname{supp} \psi_{i}\right\} \leq M .
$$

Take $m^{\prime} \geq 1$ such that $m^{\prime}\left(\Theta-\frac{n(q-1)}{p}\left(1-\frac{p}{q}\right)\right) \geq 1$.

Suppose $F(x, z)$ satisfies $(\mathrm{H} 1)$ and $(\mathrm{H} 2)$. Let $u \in W^{1, p}(\Omega)$. Suppose that there are functions $u_{\varepsilon}^{i} \in W^{1, p}\left(\Omega_{i}\right), \varepsilon_{1}>0$ and $C=C\left(\|u\|_{W^{1, p}(\Omega)}\right)$ such that for each $i \in I$, $\varepsilon \in\left(0, \varepsilon_{1}\right)$,

1. $u_{\varepsilon}^{i} \rightarrow u$ in $W^{1, p}\left(\Omega_{i}\right)$ as $\varepsilon \rightarrow 0$ and

$$
\begin{aligned}
\left\|u_{\varepsilon}^{i}-u\right\|_{L^{q}\left(\Omega_{i}\right)} & \lesssim C\left\|D \psi_{i}\right\|_{L^{\infty}(\Omega)}^{-m \Theta} \varepsilon \\
\left\|D u_{\varepsilon}^{i}\right\|_{L^{q}\left(\Omega_{i}\right)} & \lesssim C\left(\varepsilon\left\|D \psi_{i}\right\|_{L^{\infty}(\Omega)}^{-m}\right)^{-\frac{n}{p}\left(1-\frac{p}{q}\right)} .
\end{aligned}
$$

for some $m \geq m^{\prime}$. Then $u_{\varepsilon}=\sum_{i \in I} u_{\varepsilon}^{i} \psi_{i} \rightarrow u$ in $W^{1, p}(\Omega)$ as $\varepsilon \rightarrow 0$ and

$$
\int_{\Omega} F\left(x, D u_{\varepsilon}\right) \mathrm{d} x \lesssim c\left(\|u\|_{W^{1, p}(\Omega)}\right)+\int_{\Omega} F\left(x, \sum_{i \in I} D u_{\varepsilon}^{i} \psi_{i}\right) \mathrm{d} x .
$$


Proof We remark that by the restriction on $q, m^{\prime}$ is well-defined. Let $\varepsilon \in\left(0, \varepsilon_{0}\right)$. We first show that $u_{\varepsilon} \in W^{1, p}(\Omega)$. By Sobolev embedding, (26) and strong convergence of $u_{\varepsilon}^{i}$, we see that $u_{\varepsilon} \rightarrow u$ in $L^{p}(\Omega)$. Thus we focus on

$$
D u_{\varepsilon}=\sum_{i \in I} D u_{\varepsilon}^{i} \psi_{i}+\sum_{i \in I} u_{\varepsilon}^{i} \otimes D \psi_{i}=A_{1}+A_{2} .
$$

Now due to (26), $A_{1} \in L^{p}(\Omega)$. For $A_{2}$ note using the fact that $\sum_{i \in I} D \psi_{i}=0$, Lemma 2 and again (26),

$$
\left\|A_{2}\right\|_{L^{q}(\Omega)} \lesssim C\left\|\sum_{i \in I} \varepsilon^{\Theta}\right\| D \psi_{i}\left\|_{L^{\infty}(\Omega)}^{-m \Theta+1} 1_{\operatorname{supp} \psi_{i}}\right\|_{L^{q}(\Omega)} \lesssim C \varepsilon^{\Theta} .
$$

To obtain the last inequality we also noted the choice of $m^{\prime}$. Hence $u_{\varepsilon} \rightarrow u$ in $W^{1, p}(\Omega)$. Using (2), we find using also the bound on $A_{2}$,

$$
\begin{aligned}
& \int_{\Omega} F\left(x, D u_{\varepsilon}\right) \mathrm{d} x \lesssim \int_{\Omega} F\left(x, A_{1}\right)+\left|A_{2}\right|\left(1+\left|A_{1}\right|^{q-1}+\left|A_{2}\right|^{q-1}\right) \mathrm{d} x \\
& \quad \lesssim 1+C+\int_{\Omega} F\left(x, A_{1}\right) \mathrm{d} x+\int_{\Omega}\left|A_{1}\right|^{q-1}\left|A_{2}\right| \mathrm{d} x .
\end{aligned}
$$

Set for $j \in I, I_{j}=\left\{i \in I: \operatorname{supp} \psi_{i} \cap \operatorname{supp} \psi_{j} \neq \emptyset\right\}$. Then using Hölder's inequality and Lemma 2

$$
\begin{aligned}
& \int_{\Omega}\left|A_{1}\right|^{q-1}\left|A_{2}\right| \mathrm{d} x \leq \sum_{j \in I} \int_{\Omega_{j}}\left|A_{2}\right|\left|D u_{\varepsilon}^{j}\right|^{q-1} \mathrm{~d} x \\
& \quad \lesssim C^{q} \sum_{j \in I}\left(\varepsilon\left\|D \psi_{j}\right\|_{L^{q}\left(\Omega_{j}\right)}^{-m}\right)^{-\frac{n}{p}\left(1-\frac{p}{q}\right)} \sum_{I_{j}} \varepsilon^{\Theta}\left\|D \psi_{i}\right\|_{L^{\infty}\left(\Omega_{i}\right)}^{-m \Theta+1}\left\|1_{\psi_{i}}\right\|_{L^{q}(\Omega)} \\
& \lesssim C^{q} \varepsilon^{\tau} \sum_{i \in I}\left\|D \psi_{i}\right\|_{L^{\infty}\left(\Omega_{i}\right)}^{-m \tau+1}\left\|1_{\psi_{i}}\right\|_{L^{q}(\Omega)}
\end{aligned}
$$

with $\tau=\Theta-\frac{n(q-1)}{p}\left(1-\frac{p}{q}\right)>0$ by our restriction on $q$. By our choice of $m^{\prime}$ and (26), we conclude that the right-hand side is bounded uniformly in $\varepsilon$. This concludes the proof.

We next specialise to a specific choice of $u_{\varepsilon}^{i}$ and deal with convergence of the term $\left.\int_{\Omega} F\left(x, \sum_{i \in I} D u_{\varepsilon}^{i} \psi_{i}\right)\right) \mathrm{d} x$.

Lemma 14 Let $\Omega$ be a domain. Suppose $1<p \leq q<\frac{(n+\alpha) p}{n}$. Choose $m^{\prime} \geq 1$ such that $m^{\prime}\left(\Theta-\frac{n(q-1)}{p}\left(1-\frac{p}{q}\right)\right) \geq 1$. Given $u \in W^{1, p}(\Omega)$, any $\varepsilon \in\left(0,1 /(1+1 / 6)^{n}\right)$ and $m \geq 1$ write

$$
u_{\varepsilon}=\sum_{i \in I} u \star \phi_{\varepsilon \delta_{i}} \psi_{i} \quad \text { with } \delta_{i}=\left|K_{i}\right|^{\frac{m}{n}}
$$

Here $\left\{K_{i}\right\}_{i \in I}$, a WB-covering of $\Omega$ and $\left\{\psi_{i}\right\}_{i \in I}$ the partition of unity associated to this covering by Theorem 5. Assume that $F(x, \cdot)$ satisfies $(\mathrm{H} 1)-(\mathrm{H} 3)$ and $(\mathrm{H} 4)$. Then if $m>m^{\prime}$, up to passing to a subsequence if necessary, $u_{\varepsilon} \in W_{u}^{1, p}(\Omega) \cap W_{\operatorname{loc}}^{1, q}(\Omega), u_{\varepsilon} \rightarrow u$ in $W^{1, p}(\Omega)$ and

$$
\int_{\Omega} F\left(x, D u_{\varepsilon}\right) \mathrm{d} x \rightarrow \int_{\Omega} F(x, D u) \mathrm{d} x \quad \text { as } \varepsilon \searrow 0 .
$$


Proof The well-definedness of $u_{\varepsilon}$ is ensured by Theorems 4 and 5 .

By Theorem 5 and Lemma 2 as well as our choice of $m^{\prime}$, we may apply Lemma 13 to find that $u_{\varepsilon} \in W^{1, p}(\Omega)$ and $u_{\varepsilon} \rightarrow u$ in $W^{1, p}(\Omega)$ as $\varepsilon \searrow 0$. By (10) and Lemma 2 it is clear that $u_{\varepsilon} \in W_{\mathrm{loc}}^{1, q}(\Omega)$. We turn to the boundary behaviour of $u_{\varepsilon}$. By a density argument we may assume that $u \in C(\bar{\Omega})$. Note for $x \in \Omega$,

$$
\left|u_{\varepsilon}(x)-u(x)\right| \leq \sum_{i \in I: x_{n} \in K_{i}}\left|u \star \rho_{\varepsilon \delta_{i}}(x)-u(x)\right| \psi_{i} \leq M \max _{y \in B_{r}(x)}|u(y)-u(x)|
$$

where $r=\max \left\{\delta_{i}: i \in I, x \in K_{i}\right\}$. But if $x \in K_{i}$, then $\delta_{i} \lesssim\left|K_{i}\right|^{\frac{1}{n}} \sim d(x, \partial \Omega)$. It follows that $u_{\varepsilon} \in W_{u}^{1, p}(\Omega)$.

Finally, again by Lemma 13,

$$
\int_{\Omega} F\left(x, D u_{\varepsilon}\right) \mathrm{d} x \lesssim c\left(\|u\|_{W^{1, p}(\Omega)}\right)+\int_{\Omega} F\left(x, \sum_{i \in I} D u \star \phi_{\varepsilon \delta_{i}} \psi_{i}\right) \mathrm{d} x .
$$

For $\varepsilon<\varepsilon_{0}\left(\max _{i \in I}\left|K_{i}\right|^{\frac{1}{n}}\right)^{-1}$, we find using Jensen's inequality, (10), Lemma 12, Lemma 2 and the dominated convergence theorem,

$$
\begin{aligned}
& \int_{\Omega} F\left(x, \sum_{i \in I} D u \star \phi_{\varepsilon} \delta_{i} \psi_{i}\right) \leq \sum_{i \in I} \int_{\Omega} \int_{B_{1}} F\left(x, D u\left(x-\varepsilon \delta_{i} y\right)\right) \phi(y) \mathrm{d} y \psi_{i} \mathrm{~d} x \\
& \lesssim 1+\sum_{i \in I} \int_{\Omega}\left(F(\cdot, D u(\cdot)) \star \phi_{\varepsilon \delta_{i}}\right)(x) \psi_{i} \mathrm{~d} x \\
& \stackrel{\varepsilon \rightarrow 0}{\longrightarrow} 1+\sum_{i \in I} \int_{\Omega} F(x, D u) \psi_{i} \mathrm{~d} x=1+\mathscr{F}(D u)<\infty .
\end{aligned}
$$

An application of a version of the dominated convergence theorem concludes the proof.

\subsection{Proof of Theorem 2}

We are now able to prove Theorem 2 .

Proof of Theorem 2 Let $u \in W_{g}^{1, p}(\Omega)$. Let $\Omega^{s}, \Psi_{s}, \hat{g}$ be as constructed in Lemma 6. Extend $u$ by $\hat{g}$ to $\Omega^{s}$, still denoting the extension by $u$. Define

$$
\begin{aligned}
F^{s}(x, z) & =F\left(\Psi_{s}^{-1}(x), z D \Psi_{s}\left(\Psi_{s}^{-1}(x)\right)\right) \quad \mathscr{F}^{s}(u)=\int_{\Omega} F^{s}(x, D u)-f \cdot u \mathrm{~d} x \\
u^{s}(x) & =u\left(\Psi_{s}^{-1}(x)\right) .
\end{aligned}
$$

By Lemma $4, u \in W^{1, p}\left(\Omega^{s}\right)$. Since $\left|D \Psi_{s}-\mathrm{Id}\right| \rightarrow 0$ uniformly in $\Omega$, we find $u^{s} \rightarrow u \in W^{1, p}(\Omega)$. With the change of coordinates $\tilde{x}=\psi^{-1}(x)$ we may compute

$$
\begin{aligned}
\int_{\Omega} F^{s}\left(x, D u^{s}\right) \mathrm{d} x & =\int_{\Omega} F\left(\Psi_{s}^{-1}(x), D u\left(\Psi_{s}^{-1}(x)\right) D \Psi_{s}^{-1}(x) D \Psi_{s}\left(\Psi_{s}^{-1}(x)\right)\right) \\
& =\int_{\Omega} F\left(\Psi_{s}^{-1}(x), D u\left(\Psi_{s}^{-1}(x)\right) \mathrm{d} x\right. \\
& =\int_{\Omega} F(\tilde{x}, D u(\tilde{x})) J_{\Psi_{s}} \mathrm{~d} \tilde{x}+\int_{\Omega^{s} \backslash \Omega} F(\tilde{x}, D u(\tilde{x})) J_{\psi_{s}} \mathrm{~d} \tilde{x}
\end{aligned}
$$




$$
\rightarrow \int_{\Omega} F(D u) \mathrm{d} x
$$

as $s \nearrow 1$, using (H2) and absolute continuity of the integral since we have $u \in W^{1, q}\left(\Omega^{s} \backslash \Omega\right)$.

We check that there is $s_{0}>0$ such that $F^{s}(x, z)$ satisfies $(\mathrm{H} 1)-(\mathrm{H} 3)$ and $(\mathrm{H} 4)$ with constants independent of $s$ for $s \in\left(0, s_{0}\right)$. Using the properties of $\Psi_{s}$ obtained from Lemma 6 straightforward computations show that (H1)-(H3) hold if $s \in\left(0, s_{0}\right)$ for some $s_{0}>0$. Supoose (H4) holds for $F(x, z)$. Let $\varepsilon \in\left(0, \varepsilon_{0}\right)$. Choosing $\tau$ sufficiently small, we can ensure that the implication $|x-y| \leq \tau \Rightarrow\left|\psi_{s}^{-1}(x)-\psi_{s}^{-1}(y)\right|<\varepsilon$ holds for all $s \in\left(0, s_{0}\right)$. Thus,

$$
\min _{x \in \overline{B_{\tau}\left(x_{0}\right) \cap \Omega}} F^{s}(x, z)=\min _{x \in B_{\varepsilon}\left(\Psi_{s}^{-1}\left(x_{0}\right)\right) \cap \Omega} F\left(x, z D \Psi_{s}\left(\Psi_{s}^{-1}(x)\right)\right)
$$

and so $F^{s}(x, z)$ satisfies (H4).

Let now $u$ be a pointwise minimiser of $\mathscr{F}(\cdot)$ in the class $W_{g}^{1, p}(\Omega)$. Using our observations on $u^{s}$, Lemma 14 and a diagonal subsequence argument we find a sequence $u_{k} \in W_{g}^{1, q}(\Omega)$ such that $u_{k} \rightarrow u$ weakly in $W^{1, p}(\Omega)$ and in addition

$$
\int_{\Omega} F^{1 / k}\left(x, D u_{k}\right) \mathrm{d} x \rightarrow \int_{\Omega} F(x, D u) \mathrm{d} x .
$$

Fix $0 \leq \beta<\alpha$. Since $F^{s}(x, z)$ satisfies (H1)-(H3) we may apply Lemma 11 to find that the pointwise minimisers $v_{k}$ of

$$
\mathscr{F}_{\varepsilon_{k}}^{1 / k}(v)=\int_{\Omega} F^{1 / k}(x, D v)+\varepsilon_{k}|D v|^{q}-f \cdot v \mathrm{~d} x
$$

satisfy for some $\gamma>0$ the apriori estimate

$$
\left\|v_{k}\right\|_{W^{1, \frac{n p}{n-\beta}(\Omega)}} \lesssim\left(1+\mathscr{F}_{\varepsilon_{k}}^{1 / k}\left(v_{k}\right)+\|g\|_{W^{1+\max \left(\alpha, \frac{1}{q}\right), q}(\Omega)}+\|f\|_{L^{q^{\prime}(\Omega)}}\right)^{\gamma} .
$$

Here $\varepsilon_{k}=\left(1+\varepsilon^{-1}+\left\|D u_{k}\right\|_{L^{q}(\Omega)}\right)^{-1}$. Using the minimality of $v_{k}$ and then the choice of $u_{k}$ and $\varepsilon_{k}$, we deduce

$$
\begin{aligned}
\left\|v_{k}\right\|_{W}^{1, \frac{n p}{n-\beta}(\Omega)} & \lesssim\left(1+\mathscr{F}_{\varepsilon_{k}}^{1 / k}\left(u_{k}\right)+\|g\|_{W^{1+\max \left(\alpha, \frac{1}{q}\right), q}(\Omega)}+\|f\|_{L^{q^{\prime}}(\Omega)}\right)^{\gamma} \\
& \lesssim\left(1+\mathscr{F}(u)+\|g\|_{W^{1+\max \left(\alpha, \frac{1}{q}\right), q}(\Omega)}+\|f\|_{L^{q^{\prime}(\Omega)}}\right)^{\gamma} .
\end{aligned}
$$

In particular we note that the estimate is independent of $s$ and $\varepsilon$. Choosing now $\beta$ sufficiently large that we obtain an estimate in $W^{1, q}(\Omega)$, we may extract a subsequence of $v_{k}$ converging weakly in $W^{1, q}(\Omega)$ to some $v$ and by weak lower semicontinuity of norms, $v \in W^{1, q}(\Omega)$. Moreover by weak semicontinuity and minimality of $v_{k}$ in the class $W_{g}^{1, q}(\Omega)$,

$$
\begin{aligned}
\mathscr{F}(v) & =\lim _{k \rightarrow \infty} \int_{\Omega} F^{1 / k}(x, D v)-f \cdot v \mathrm{~d} x \leq \lim _{k \rightarrow \infty} \mathscr{F}_{\varepsilon_{k}}^{1 / k}\left(x, v_{k}\right) \\
& \leq \lim _{k \rightarrow \infty} \mathscr{F}_{\varepsilon_{k}}^{1 / k}\left(u_{k}\right)=\mathscr{F}(u) .
\end{aligned}
$$

By minimality of $u$ in the class $W_{g}^{1, p}(\Omega)$, we conclude $\mathscr{F}(v)=\mathscr{F}(u)$. But then by convexity of $\mathscr{F}(\cdot)$ it follows that $v=u$, concluding the proof.

We briefly comment on how to prove Corollary 1. 
Proof of Corollary 1 Once Theorem 2 is available, there is no problem in making sense of the Euler-Lagrange equation for $u$. Hence we may repeat the arguments of Lemma 11 with $\mathscr{F}_{\varepsilon}(\cdot)$ and $v_{\varepsilon}+g$ replaced by $\mathscr{F}(\cdot)$ and $u$ respectively. The equivalent estimate to (21) and Theorem 3 then give the result. In the case of Theorem 1 using Lemma 10 the same argument applies.

\subsection{Proof of Proposition 1}

We intend to use Lemma 5 to reduce to the case of a Lipschitz domain. Thus we focus first on the case of improving the regularity near the boundary for the sequence $\left(u_{\varepsilon}\right)$ from Lemma 14 in the case of a star-shaped domain.

Lemma 15 Let $1<p \leq q<\left(1+\frac{\alpha}{(\alpha+1) n}\right) p$. Suppose $\Omega$ is a strongly star-shaped Lipschitz domain. Let $\gamma^{\prime} \Subset \gamma \subset \partial \Omega$ where $\gamma^{\prime}$ and $\gamma$ are connected. Suppose $\Omega^{\prime} \subset \Omega$ with $\partial \Omega^{\prime} \cap$ $\partial \Omega=\gamma^{\prime}$ and such that if $N_{\gamma^{\prime}}$ is a neighbourhood of $\gamma^{\prime}$, then $\Omega^{\prime} \backslash N_{\gamma^{\prime}} \Subset \Omega$. If $u \in$ $W^{1, p}(\Omega) \cap W^{1, p}(\partial \Omega) \cap W^{1, q}(\gamma)$, then there is $\left(u_{n}\right) \subset W_{u}^{1, p}(\Omega) \cap W^{1, q}\left(\Omega^{\prime}\right)$ such that $u_{n} \rightarrow u$ in $W^{1, p}(\Omega)$ and $\int_{\Omega^{\prime}} F\left(x, D u_{n}\right) \mathrm{d} x \rightarrow \int_{\Omega^{\prime}} F(x, D u) \mathrm{d} x$.

Proof Without loss of generality assume that $\Omega$ is strongly star-shaped with respect to 0 . Let $u \in W^{1, p}(\Omega) \cap W^{1, p}(\partial \Omega) \cap W^{1, q}(\gamma)$. Consider the homogeneous extension of $u$ to $\mathbb{R}^{n}$ by setting

$$
u(x)= \begin{cases}u(x) & \text { if } x \in \Omega \\ 1 / \lambda^{\prime} u\left(\lambda^{\prime} x\right) & \text { if } x \notin \Omega \text { where } \lambda^{\prime}=\sup \{\lambda>0: \lambda x \in \bar{\Omega}\} .\end{cases}
$$

For $\frac{1}{2}<s<x$ we define $u^{s}(x)=s u(x / s)$. It is easy to check that $u_{s} \in W_{u}^{1, p}(\Omega)$ and $u_{s} \rightarrow u$ in $W^{1, p}(\Omega)$ as $s \nearrow 1$. Let $\left\{K_{i}\right\}_{i \in I}$ be a WB-covering of $\Omega$ and $\left\{\psi_{i}\right\}_{i \in I}$ the partition of unity associated to this covering by Theorem 5. The existence of $\left\{K_{i}\right\}_{i \in I}$ and $\left\{\psi_{i}\right\}_{i \in I}$ is ensured by Theorems 4 and 5 .

Take $m \geq 1$ such that $m\left(\Theta-\frac{n(q-1)}{p}\left(1-\frac{p}{q}\right)\right)>1$. Define with $\delta_{i}=\left|K_{i}\right|^{\frac{m}{n}}$,

$$
u_{\varepsilon}^{s}=\sum_{i \in I} u^{s} \star \phi_{\varepsilon \delta_{i}} \psi_{i} \quad u_{\varepsilon}=\sum_{i \in I} u \star \phi_{\varepsilon \delta_{i}} \psi_{i} .
$$

Note that for $s, \varepsilon$ sufficiently small, $u_{\varepsilon}^{s}$ is well-defined. By Theorem 5 and Lemma 2, we may apply Lemma 13 to $u_{\varepsilon}^{s}$ in order to see that $\left(u_{\varepsilon}^{s}\right) \in W_{u}^{1, p}(\Omega)$ with $u_{\varepsilon}^{s} \rightarrow u^{s}$ in $W^{1, p}(\Omega)$ as $\varepsilon \rightarrow 0$. Further by Lemma 2 noting that $u_{\varepsilon}^{s}$ is $W^{1, q}$-regular in a neighbourhood of $\gamma^{\prime}$, $u_{\varepsilon}^{s} \in W^{1, q}\left(\Omega^{\prime}\right)$. Using Lemma 13 ,

$$
\begin{aligned}
\int_{\Omega^{\prime}} F\left(x, D u_{\varepsilon}^{s}\right) \mathrm{d} x & \lesssim c\left(\left\|u^{s}\right\|_{W^{1, p}(\Omega)}\right)+\int_{\Omega^{\prime}} F\left(x, \sum_{i \in I} D u_{\varepsilon}^{s} \psi_{i}\right) \mathrm{d} x \\
& \lesssim c\left(\|u\|_{W^{1, p}(\Omega)}+\|u\|_{W^{1, p}(\partial \Omega)}\right)+\int_{\Omega^{\prime}} F\left(x, \sum_{i \in I} D u^{s} \star \phi_{\varepsilon \delta_{i}} \psi_{i}\right) .
\end{aligned}
$$

Let $1-s$ be sufficiently small that $\partial\left(s^{-1} \Omega^{\prime} \backslash \Omega\right) \subset \gamma$ and in addition for any neighbourhood $N_{\gamma}$ of $\gamma,\left(s^{-1} \Omega^{\prime} \cap \Omega\right) \backslash N_{\gamma} \Subset \Omega$. Then using the change of coordinates $x \rightarrow s x$,

$$
\int_{\Omega^{\prime}} F\left(x, \sum_{i \in I} D u_{\varepsilon}^{s} \psi_{i}\right) \mathrm{d} x=s^{-n} \int_{s^{-1} \Omega^{\prime}} F(s x, A) \mathrm{d} x
$$




$$
\leq s^{-n} \int_{s^{-1} \Omega^{\prime} \backslash \Omega} F(s x, A) \mathrm{d} x+s^{-n} \int_{s^{-1} \Omega^{\prime} \cap \Omega} F(s x, A) \mathrm{d} x
$$

with $A=\sum_{i \in I}\left(D u(\cdot) \star \phi_{\varepsilon \delta_{i}}\right)(x) \psi_{i}(s x)$. Now since $\|u\|_{W^{1, q}\left(s^{-1} \Omega^{\prime} \backslash \Omega\right)} \lesssim\|u\|_{W^{1, q}(\gamma)}$, we may use (H2), Lemma 2 and (10) to see that

$$
s^{-n} \int_{s^{-1} \Omega^{\prime} \backslash \Omega} F(s x, A) \mathrm{d} x \lesssim s^{-n} \int_{s^{-1} \Omega^{\prime} \backslash \Omega} 1+|A|^{q} \mathrm{~d} x \lesssim 1+\|u\|_{W^{1, q}(\gamma)}
$$

independently of $s$ and $\varepsilon$. Note that if $x \in \operatorname{supp} \psi_{i}(s \cdot) \cap \Omega$, then we have $\left|K_{i}\right|^{\frac{1}{n}} \sim d(x, \partial \Omega) \gtrsim(1-s)$ and so $\delta_{i} \gtrsim(1-s)^{m}$. Thus we find for some sufficiently small $c>0$ and using Lemma 2 as well as (H3),

$$
\begin{aligned}
& \int_{s^{-1} \Omega^{\prime} \cap \Omega}(1-s)^{\alpha}\left|A_{1}\right|^{q} \mathrm{~d} x \\
& \quad \lesssim(1-s)^{\alpha} \int_{s^{-1} \Omega^{\prime} \cap \Omega} \sum_{i \in I:\left|K_{i}\right|^{\frac{1}{n}} \geq c(1-s)}\left(\varepsilon \delta_{i}\right)^{-\frac{n}{p}(q-p)}\|u\|_{W^{1, p}\left(K_{i}\right)}^{p} .
\end{aligned}
$$

Recalling the definition of $\delta_{i}$, if $\alpha-m \frac{n}{p}(q-p) \geq 0$, then the right-hand side is bounded independently of $s$. Recalling the choice of $m$ we find that we need to have $q<\left(1+\frac{\alpha}{(\alpha+1) n}\right) p$.

By a version of the dominated convergence theorem we conclude since $D u_{\varepsilon}^{s} \rightarrow D u^{s}$ almost everywhere in $\Omega^{\prime}$,

$$
\int_{\Omega^{\prime}} F\left(x, D u_{\varepsilon}^{s}\right) \mathrm{d} x \rightarrow \int_{\Omega^{\prime}} F\left(x, D u_{\varepsilon}\right) \mathrm{d} x \quad \text { as } s \nearrow 1 .
$$

By Lemma 14

$$
\int_{\Omega^{\prime}} F\left(x, D u_{\varepsilon}\right) \mathrm{d} x \rightarrow \int_{\Omega^{\prime}} F(x, D u) \mathrm{d} x \quad \text { as } \varepsilon \searrow 0 .
$$

Thus by a diagonal subsequence argument, we can extract a subsequence of $\left(u_{\varepsilon}^{s}\right)$ with all the desired properties.

We can now finally prove Proposition 1.

Proof of Proposition 1 Note that the first part of Proposition 1 is a consequence of Lemmas 14 and 8.

For the second part by Lemma 8 it suffices to exhibit $\left(u_{n}\right) \subset W_{g}^{1, q}(\Omega)$ such that $u_{n} \rightarrow u$ weakly in $W^{1, p}(\Omega)$ and $\mathscr{F}\left(u_{n}\right) \rightarrow \mathscr{F}(u)$ as $n \rightarrow \infty$. By Lemma 5 we may write

$$
\Omega=\bigcup_{i=1}^{N} \Omega_{i}=\bigcup_{i=1}^{N} \omega_{i}
$$

where $\Omega_{i}$ is a strongly star-shaped Lipschitz domain, $\omega_{i} \Subset \Omega_{i}$ relative to $\Omega$ and further $\partial \omega_{i} \cap \partial \Omega \Subset \partial \Omega_{i} \cap \partial \Omega$ if $\partial \Omega_{i} \cap \Omega \neq \varnothing$. We may also assume that $u \in W^{1, p}\left(\partial \Omega_{i}\right)$ for $i=1, \ldots, N$ with

$$
\|u\|_{W^{1, p}\left(\partial \Omega_{i}\right)} \lesssim\|u\|_{W^{1, p}\left(\Omega_{i}\right)}+\|g\|_{W^{1+1 / q, q}(\Omega)} .
$$

Let $\psi_{i}$ be a partition of unity subordinate to $\omega_{i}$. By Lemma 15, there are sequences $\left(u_{n}^{i}\right) \subset W^{1, p}\left(\Omega_{i}\right) \cap W^{1, q}\left(\omega_{i}\right)$ such that $u_{n}^{i} \rightarrow u$ in $W^{1, p}(\Omega)$ and $\int_{\omega_{i}} F\left(x, D u_{n}^{i}\right) \mathrm{d} x \rightarrow$ 
$\int_{\omega_{i}} F(x, D u) \mathrm{d} x$ where $i=1, \ldots, N$. Consider

$$
u_{n}=\sum_{i=1}^{N} u_{n}^{i} \psi_{i}
$$

Then $u_{n} \in W_{g}^{1, q}(\Omega)$ and $u_{n} \rightarrow u$ in $W^{1, p}(\Omega)$. Moreover using (2), Hölder's inequality, Sobolev's embedding and Jensen's inequality,

$$
\begin{aligned}
\int_{\Omega} F\left(x, D u_{n}\right) \mathrm{d} x \lesssim & \sum_{i, j=1}^{N} \int_{\Omega}\left|u_{n}^{i} \otimes D \psi_{i}\right|\left(1+\left|u_{n}^{i} \otimes D \psi_{j}\right|+\left|D u_{n}^{j} \otimes D \psi_{j}\right|\right)^{q-1} \mathrm{~d} x \\
& +\int_{\Omega} F\left(x, \sum_{i=1}^{N} D u_{n}^{i} \psi_{i}\right) \mathrm{d} x \\
\lesssim & \sum_{i, j=1}^{n}\left\|D u_{n}^{i}\right\|_{L^{p}\left(\Omega_{i}\right)}^{q-1}\left\|D u_{n}^{j}\right\|_{L^{p}\left(\Omega_{j}\right)}^{q}+\sum_{i=1}^{N} \int_{\omega_{i}} F\left(x, D u_{n}^{i}\right) \psi_{i} \mathrm{~d} x \\
& \rightarrow \sum_{i, j=1}^{n}\left\|D u_{n}^{i}\right\|_{L^{p}\left(\Omega_{i}\right)}^{q-1}\left\|D u_{n}^{j}\right\|_{L^{p}\left(\Omega_{j}\right)}^{q}+\int_{\Omega} F(x, D u) \mathrm{d} x<\infty .
\end{aligned}
$$

To obtain the second line we used the assumption on $q$. Thus we conclude by a variant of the domianted convergence theorem that $\mathscr{F}\left(u_{n}\right) \rightarrow \mathscr{F}(u)$ as $n \rightarrow \infty$.

\section{Regularity under alternatives to assumption (H1)}

In this section we explore some alternatives to (H1) which only require minor modifications of the proofs. To be precise we consider the following assumptions: Assume one of the following holds for some $\mu \geq 0, \lambda>0$ and for all $z, w \in \mathbb{R}^{n \times m}$ and almost every $x \in \Omega$ :

$$
\begin{aligned}
& \left\{\begin{array}{c}
\lambda\left(\mu^{2}+|z|^{2}+|w|^{2}\right)^{\frac{p(x)-2}{2}}|z-w|^{2} \\
\quad \leq F(x, z)-F(z, w)-\partial_{z} F(x, w) \cdot(z-w) \\
\text { where } p(x) \in C^{0, \alpha}(\Omega) \text { and there is } \varepsilon>0 \text { such that } 1<p \leq p(x) \leq q
\end{array}\right. \\
& \left\{\begin{array}{c}
\lambda \sum_{i=1}^{n}\left(\mu_{i}^{2}+\left|z_{i}\right|^{2}+\left|w_{i}\right|^{2}\right)^{\frac{p_{i}-2}{2}}\left|z_{i}-w_{i}\right|^{2} \\
\quad \leq F(x, z)-F(z, w)-\partial_{z} F(x, w) \cdot(z-w) \\
\text { where } z_{i}=\left(z_{i}\right)_{1 \leq j \leq n}^{j}, w_{i}=\left(w_{i}\right)_{1 \leq j \leq n}^{j} \text { and } 1<p=p_{1} \leq p_{2} \leq \cdots \leq p_{n}=q
\end{array}\right.
\end{aligned}
$$

Before commencing to prove versions of Theorems 1 and 2, we remark that replacing (H1) with (H1.1) or (H1.2) also causes a change in the proof of Lemma 7: Using the notation of Lemma 7, (16) needs to be replaced by

$$
\overline{\mathscr{F}}\left(\frac{w_{1}+w_{2}}{2}\right)+\frac{v}{p}\left(\int_{\Omega}\left|D w_{1}-D w_{2}\right|^{p(x)} \mathrm{d} x\right)^{\frac{1}{p}} \leq \frac{1}{2}\left(\overline{\mathscr{F}}\left(w_{1}\right)+\overline{\mathscr{F}}\left(w_{2}\right)\right) .
$$


or

$$
\overline{\mathscr{F}}\left(\frac{w_{1}+w_{2}}{2}\right)+\frac{v}{p}\left(\int_{\Omega} \sum_{i=1}^{n}\left|D_{i} w_{1}-D_{i} w_{2}\right|^{p_{i}} \mathrm{~d} x\right)^{\frac{1}{p}} \leq \frac{1}{2}\left(\overline{\mathscr{F}}\left(w_{1}\right)+\overline{\mathscr{F}}\left(w_{2}\right)\right),
$$

respectively. Arguing as before, either estimate suffices to conclude that we have $D u_{\varepsilon} \rightarrow D u$ in $L^{p}(\Omega)$.

We then have:

Theorem 7 Suppose $g \in W^{1+\alpha, q}(\Omega)$ and $F(x, z)$ satisfies assumptions (H2), (H3) and either (H1.1) or (H1.2). Suppose $1 \leq p<q<\frac{(n+\alpha) p}{n}$. Then the conclusion of Theorem 1 still holds. If in fact $g \in W^{1+\max (\alpha, 1 / q), q}(\Omega)$ and $F(x, \cdot)$ satisfies in addition (H4), then the conclusion of Theorem 2 holds.

Proof For simplicity we only focus on the case $p \geq 2$. The case $p<2$ follows from similar considerations. Assume first that (H1.1) holds. Using the notation of Sect. 2.3, extend $p(x)$ to a $C^{0, \alpha}$-function on $B(0, R)$ with $p(x) \in[p, q]$ by setting

$$
p(x)=\inf _{y \in \Omega}\left(p(y)+\|p\|_{C^{0, \alpha}(\Omega)}|x-y|\right) .
$$

for $x \in B(0, R) \backslash \Omega$. The first change to the argument occurs in the proof of the a-priori estimate in Lemma (11). Instead of (17) we obtain:

$$
\begin{aligned}
& \mathscr{F}_{\varepsilon}(v+g)-\mathscr{F}_{\varepsilon}\left(v_{\varepsilon}+g\right) \\
& \quad \gtrsim \int_{\Omega}\left(\mu^{2}+|D v+D g|^{2}+\left|D v_{\varepsilon}+D g\right|^{2}\right)^{\frac{p(x)-2}{2}}\left|D v-D v_{\varepsilon}\right|^{2} \mathrm{~d} x,
\end{aligned}
$$

We now argue as before to conclude,

$$
\begin{aligned}
\int_{\Omega}\left|D v_{\varepsilon}\right|^{\frac{n p(x)}{n-\beta}} \mathrm{d} x \lesssim & \left(1+\frac{1}{2}\left\|v_{\varepsilon}\right\|_{W^{1, \frac{n p}{n-\beta}(\Omega)}}^{p}+C(\theta)\left\|v_{\varepsilon}\right\|_{W^{1, p}(\Omega)}^{\frac{\theta q}{(\theta q-p)}}\right. \\
& \left.+\|g\|_{W^{1+\alpha, q}(\Omega)}^{q}+\|f\|_{L^{q^{\prime}(\Omega)}}^{q^{\prime}}\right) .
\end{aligned}
$$

Noting that

$$
\int_{\Omega}\left|D v_{\varepsilon}\right|^{\frac{n p}{n-\beta}} \mathrm{d} x \lesssim \int_{\Omega}\left|D v_{\varepsilon}\right|^{\frac{n p(x)}{n-\beta}} \mathrm{d} x+1 .
$$

we finish the proof without further change.

For the proof of Theorem 2 we only need to verify that $(\mathrm{H} 1.1)$ holds for $F^{s}(x, \cdot)$. The computation is straightforward.

We now assume that (H1.2) holds. Instead of (17) we have

$$
\begin{aligned}
& \mathscr{F}_{\varepsilon}(v+g)-\mathscr{F}_{\varepsilon}\left(v_{\varepsilon}+g\right) \\
& \quad \gtrsim \int_{\Omega} \sum_{i=1}^{n}\left(\mu_{i}^{2}+\left|D v_{i}+D g_{i}\right|^{2}+\left|D v_{\varepsilon, i}+D g_{i}\right|^{2}\right)^{\frac{p_{i}-2}{2}}\left|D v_{i}-D v_{\varepsilon, i}\right|^{2} \mathrm{~d} x .
\end{aligned}
$$

Repeating the arguments given in Lemma 11 we use this to obtain

$$
\int_{\Omega} \sum_{i=1}^{n}\left|D v_{\varepsilon, i}\right|^{\frac{n p_{i}}{n-\beta}} \mathrm{d} x \lesssim\left(1+\frac{1}{2}\left\|v_{\varepsilon}\right\|_{W}^{p, \frac{n p}{n-\beta}(\Omega)}+C(\theta)\left\|v_{\varepsilon}\right\|_{W^{1, p}(\Omega)}^{\frac{\theta q}{(\theta q-p)}}\right.
$$




$$
\left.+\|g\|_{W^{1+\alpha, q}(\Omega)}^{q}+\|f\|_{L^{q^{\prime}(\Omega)}}^{q^{\prime}}\right) .
$$

The proof now concludes by the same arguments as before by noting that

$$
\int_{\Omega}\left|D v_{\varepsilon}\right|^{\frac{n p}{n-\beta}} \mathrm{d} x \lesssim 1+\int_{\Omega} \sum_{i=1}^{n}\left|D v_{\varepsilon, i}\right|^{\frac{n p_{i}(x)}{n-\beta}} \mathrm{d} x
$$

In the proof of Theorem 2 we need to verify that $(\mathrm{H} 1.2)$ holds for $F^{s}(x, \cdot)$. The computation is straightforward.

Remark 2 It is straightforward to adapt the arguments of Theorem 7 to to growth conditions that combine (H1.1) and (H1.2):

$$
\left\{\begin{array}{l}
\sum_{i=1}^{n}\left(\mu_{i}^{2}+\left|z_{i}\right|^{2}+\left|w_{i}\right|^{2}\right)^{\frac{p_{i}(x)-2}{2}}\left|z_{i}-w_{i}\right|^{2} \\
\quad \leq F(x, z)-F(z, w)-\partial_{z} F(x, w) \cdot(z-w) \\
\text { where } z_{i}=\left(z_{i}\right)_{1 \leq j \leq n}^{j}, w_{i}=\left(w_{i}\right)_{1 \leq j \leq n}^{j} \text { and } 1<p \leq p_{i}(x) \leq q .
\end{array}\right.
$$

\section{Examples}

In this section we list a number of examples to which our theory applies. We highlight in particular that we can treat the double-phase functional (iii), the anisotropic $p(x)$-Laplacian (v) as well as more general anisotropic functionals, e.g. (iv). The theory developed in this paper applies to all the functionals listed below:

(i) $\mathscr{F}_{1}(u)=\int_{\Omega} a(x) F(D u) \mathrm{d} x$, where $1 \leq a(\cdot) \leq L$,

(ii) $\mathscr{F}_{2}(u)=\int_{\Omega} \sum_{i=1}^{n} a_{i}(x) F_{i}\left(D_{i} u\right) \mathrm{d} x$, where $1 \leq a_{i}(\cdot) \leq L$,

(iii) $\mathscr{F}_{3}(u)=\int_{\Omega}|D u|^{p}+a(x)|D u|^{q} \mathrm{~d} x$ where $0 \leq a(x) \in C^{0, \alpha}(\Omega)$,

(iv) $\mathscr{F}_{4}(u)=\int_{\Omega}|D u|^{p}+\left|a_{\alpha, \beta}^{i, j}(x) D_{i} u^{\alpha} D_{j} u^{\beta}\right|^{\frac{q}{2}} \mathrm{~d} x$ where $a_{\alpha, \beta}^{i, j}(\cdot) \in C^{0, \alpha}(\Omega)$ and for all $x \in \Omega$ and $\xi \in \mathbb{R}^{n \times m}$,

$$
\lambda(x)|\xi|^{2} \leq a_{\alpha, \beta}^{i, j} \xi_{i}^{\alpha} \xi_{j}^{\beta} \leq L \lambda(x)|\xi|^{2}
$$

with $L \geq 1$ and $0 \leq \lambda(\cdot) \in C^{0, \alpha}(\Omega)$. where $0 \leq a_{i}(\cdot) \in C^{0, \alpha}(\Omega)$, and further $1<p \leq p_{i} \leq q$,

(v) $\mathscr{F}_{5}(u)=\int_{\Omega} \sum_{i=1}^{n}\left|D_{i} u\right|^{p_{i}(x)} \mathrm{d} x$, where $p \leq p_{i}(x) \leq q$ and $p_{i}(x) \in C^{0, \alpha}(\Omega)$,

(vi) $\mathscr{F}_{6}(u)=\int_{\Omega}|D u|^{p(x)} \log (1+|D u|) \mathrm{d} x$, where $1<p \leq p(x) \leq q$,

(vii) $\mathscr{F}_{7}(u)=\int_{\Omega}|D u|^{q}+a(x) \max \left(\left|D_{n} u\right|, 0\right) \mathrm{d} x$ where $q>2,0 \leq a(\cdot) \in C^{0, \alpha}(\Omega)$,

(viii) $\mathscr{F}_{8}(u)=\int_{\Omega} F(x, D u) \mathrm{d} x$ where $F(x, z)=h(a(x), z)$ where

(i) $t \rightarrow h(t, z)$ is increasing

(ii) $h(x, z)$ is convex in the second argument

(iii) $a(x) \in C(\bar{\Omega})$

(iv) $F(x, z)$ satisfies (H1)-(H3) (or one of (H1.1), (H1.2) instead of (H1))

The first five examples are standard. (vi) has been studied as a model case (with $p(x)=$ const $>1$ ) in [37]. (viii) is inspired by [40], whereas the last example is taken from [18].

Before making two observations that are helpful to verify that the results of this paper apply to minimisers of these examples, let us extract the precise statement for the proof of which we use (H4). 
Corollary 2 Suppose $\Omega$ is a $C^{1, \alpha}$-domain. Suppose $g \in W^{1+\max \left(\alpha, \frac{1}{q}\right), q}(\Omega)$. Assume $\mathscr{F}(\cdot)$ satisfies (H1)-(H3) with $1<p \leq q<\frac{(n+\alpha) p}{n}$. Suppose $u$ is a pointwise minimiser of $\mathscr{F}(\cdot)$ in the class $\in W_{g}^{1, p}(\Omega)$ and one of the following holds:

1. There is a sequence $u_{k} \in W_{g}^{1, p}(\Omega) \cap W_{\text {loc }}^{1, q}(\Omega)$ such that $u_{k} \rightarrow u$ weakly in $W^{1, p}(\Omega)$ and $\mathscr{F}\left(u_{k}\right) \rightarrow \mathscr{F}(u)$ as $k \rightarrow \infty$.

2. There is $\varepsilon_{0}>0$ such that for $\varepsilon \in\left(0, \varepsilon_{0}\right)$,

$$
F\left(x, D u \star \phi_{\varepsilon}\right) \lesssim 1+\left(F(\cdot, D u(\cdot)) \star \phi_{\varepsilon}\right)(x) .
$$

Then $u \in W^{1, q}(\Omega)$.

Proof Item (i) is precisely the conclusion from Lemma 8 we need in order to run the proof of Theorem 2. Item (ii) is the conclusion of Lemma 12 which is the only place (H4) is used. Hence the proof of Theorem 2 remains unchanged.

We highlight two ways of combining functionals for which the assumptions of Corollary 2 hold. First, if each $F_{i}(x, z), i \in\{1, \ldots, N\}$ for some $N \in \mathbb{N}$ satisfies assumption (ii) of Corollary 2, then $\sum_{i} F_{i}(x, z)$ also satisfies the assumption.

Second, assume $G(x, z)$ satisfies $(\mathrm{H} 1)-(\mathrm{H} 3)$ and (i) in Corollary 2. Consider $F(x, z)$ and suppose that

$$
G(x, z)-1 \lesssim F(x, z) \lesssim G(x, z)+1 .
$$

Then from Lemma 8 applied to $G(x, z)$ there is $u_{\varepsilon} \in W^{1, p}(\Omega) \cap W_{\text {loc }}^{1, q}(\Omega)$ such that $u_{\varepsilon} \rightarrow u$ weakly in $W^{1, p}(\Omega)$ and $\int_{\Omega} G\left(x, D u_{\varepsilon}\right) \mathrm{d} x \rightarrow \int_{\Omega} G(x, D u) \mathrm{d} x$. Thus by a version of the dominated convergence theorem and (31) we conclude $\int_{\Omega} F\left(x, D u_{\varepsilon}\right) \mathrm{d} x \rightarrow \int_{\Omega} F(x, D u) \mathrm{d} x$. Hence $F(x, z)$ also satisfies assumption (i) of Corollary 2.

Acknowledgements L.K. was supported by the Engineering and Physical Sciences Research Council [EP/L015811/1]. L.K. was supported by the EPSRC (EP/L015811/1).

Open Access This article is licensed under a Creative Commons Attribution 4.0 International License, which permits use, sharing, adaptation, distribution and reproduction in any medium or format, as long as you give appropriate credit to the original author(s) and the source, provide a link to the Creative Commons licence, and indicate if changes were made. The images or other third party material in this article are included in the article's Creative Commons licence, unless indicated otherwise in a credit line to the material. If material is not included in the article's Creative Commons licence and your intended use is not permitted by statutory regulation or exceeds the permitted use, you will need to obtain permission directly from the copyright holder. To view a copy of this licence, visit http://creativecommons.org/licenses/by/4.0/.

\section{References}

1. Acerbi, E., Bouchitté, G., Fonseca, I.: Relaxation of convex functionals: the gap problem. Ann. l'Institut Henri Poincare Anal. Non Lineare. 20(3), 359-390 (2003)

2. Acerbi, E., Fusco, N.: Partial regularity under anisotropic (p, q) growth conditions. J. Differ. Equ. 107, 46-67 (1994)

3. Balci, A.K., Diening, L., Surnachev, M.: New Examples on Lavrentiev Gap Using Fractals. arXiv:abs/1906.04639 (2020)

4. Baroni, P., Colombo, M., Mingione, G.: Regularity for general functionals with double phase. Calc. Var. Partial Differ. Equ. 57, 62 (2018)

5. Bögelein, V., Duzaar, F., Marcellini, P.: Parabolic equations with $(p, q)$-growth: a variational approach. Arch. Ration. Mech. Anal. 210, 219-267 (2013) 
6. Breit, D.: New regularity theorems for non-autonomous variational integrals with $(p, q)$-growth. Calc. Var. 44, 101-129 (2012)

7. Bulíček, M., Maringová, E., Stroffolini, B., Verde, A.: A boundary regularity results for minimizers of variational integrals with nonstandard growth. Nonlinear Anal. 177(A), 153-168 (2018)

8. Buttazo, G., Belloni, M.: A survey of old and recent results about the gap phenomenon in the calculus of variations. Recent Dev. Well Posed Var. Probl. Math. Appl. 331, 1-27 (1995)

9. Buttazo, G., Mizel, V.: Interpretation of the Lavrentiev phenomenon by relaxation. J. Funct. Anal. 2, 434-460 (1992)

10. Byun, S.-S., Oh, J.: Global gradient estimates for non-uniformly elliptic equations. Calc. Var. 56, 46 (2017)

11. Carbone, L.: Unbounded Functionals in the Calculus of Variations. Routledge, London (2019)

12. Carozza, M., Kristensen, J., Passarelli di Napoli, A.: Higher differentiability of minimizers of convex variational integrals. Ann. l'Institut Henri Poincare Anal. Non Lineare 28, 395-411 (2011)

13. Carozza, M., Kristensen, J., Passarelli di Napoli, A.: Regularity of minimisers of autonomous convex variational integrals. Ann. della Scu. Norm. Sup. di Pisa 13, 4 (2013)

14. Carozza, M., Kristensen, J., Passarelli di Napoli, A.: A trace preserving operator and applications. J. Math. Anal. Appl. 124170 (2020)

15. de Filippis, C., Koch, L., Kristensen, J.: Regularity in relaxed convex problems (2020) (in Prep)

16. de Filippis, C., Minigione, G.: A borderline case of Calderón-Zygmund estimates for non-uniformly elliptic problems. Algebr. i Anal. 31(3), 82-115 (2019)

17. Diening, L., Hästö, P., Harjulehto, P., Ruzicka, M.: Lebesgue and Sobolev Spaces with Variable Exponents. Springer, Berlin (2011)

18. Esposito, A., Leonetti, F., Petricca, P.V.: Absence of Lavrentiev gap for non-autonomous functionals with (p, q)-growth. Adv. Nonlinear Anal. 8(1), 73-78 (2019)

19. Esposito, L., Leonetti, F., Mingione, G.: Sharp regularity for functionals with (p, q) growth. J. Differ. Equ. 204, 5-55 (2004)

20. Evans, L.C.: Partial Differential Equations, 2nd edn. American Mathematical Society, Providence (2015)

21. Evans, L.C., Gariepy, R.F.: Measure Theory and Fine Properties of Functions. CRC Press, Boca Raton (1992)

22. Fonseca, I., Malý, J.: Relaxation of multiple integrals below the growth exponent for the energy density. Ann. l'Institut Henri Poincare Anal. Non Lineare 14, 309-338 (1997)

23. Fonseca, I., Malý, J.: From Jacobian to Hessian: distributional form and relaxation. Riv. Mat. Univ. Parma 4(4), 45-74 (2005)

24. Fonseca, I., Malý, J., Mingione, G.: Scalar minimizers with fractal singular sets. Arch. Ration. Mech. Anal. 172, 295-307 (2004)

25. Foss, M.: On Lavrentiev's phenomenon. PhD thesis, Carnegie Mellon University (2001)

26. Foss, M.: The Lavrentiev gaph phenomenon in nonlinear elasticity. Arch. Ration. Mech. Anal. 167, 336-365 (2003)

27. Giaquinta, M.: Growth conditions and regularity, a counterexample. Manuscripta Math. 59, 245-248 (1987)

28. Giaquinta, M., Giusti, E.: Differentiability of minima of nondifferentiable functionals. Invent. Math. 72, 285-298 (1983)

29. Giusti, E.: Direct Methods in the Calculus of Variations. WORLD SCIENTIFIC, Singapore (2003)

30. Grisvard, P.: Elliptic problems in nonsmooth domains. Rech. Mathématiques Appliquées, vol 22. Society for Industrial and Applied Mathematics, University City, Philadelphia (1992)

31. Hirsch, J., Schäffner, M.: Growth conditions and regularity, an optimal local boundedness result. Commun. Contemp. Math. textbf23, 2050029 (2020)

32. Hong, M.C.: Some remarks on the minimizers of variational integrals with (p, q) growth conditions. J. Differ. Equ. 6(7), 91-101 (1992)

33. Kislyakov, S., Kruglyak, N.: Stability of approximation under singular integrals, and Caldéron-Zygmund type decompositions. PDMI Preprint (2005)

34. Kislyakov, S., Kruglyak, N.: Extremal problems in interpolation theory, Whitney-Besicovitch coverings and singular integrals. Monogr. Mat. 74, 663-714 (2013)

35. Lavrentiev, M.: Sur quelques problème du calcul des variations. Ann. di Mat. Pura Appl. 4, 7-28 (1926)

36. Marcellini, P.: Regularity of minimizers of integrals of the calculus of variations with non-standard growth conditions. Arch. Ration. Mech. Anal. 105, 267-284 (1989)

37. Marcellini, P.: Regularity and existence of solutions of elliptic equations with p, q-growth conditions. J. Differ. Equ. 90, 1-30 (1991)

38. Mingione, G.: Regularity of minima: an invitation to the dark side of the calculus of variations. Appl. Math. 51(4), 355-426 (2006) 
39. Passarelli di Napoli, A., Siepe, F.: A regularity result for a class of anisotropic systems. Rend. Istit. Mat. Univ. Trieste 28, 13-31 (1996)

40. Qi, T.: Regularity of minimizers of non-isotropic integrals of the calculus of variations. Ann. di Mat. Pura Appl. 164(4), 77-87 (1993)

41. Rădulescu, V.D., Repovš, D.D.: Partial Differential Equations with Variable Exponents. CRC Press, Boca Raton (2015)

42. Rychkov, V.S.: On restrictions and extensions of the Besov and Triebel-Lezorkin spaces with respect to Lipschitz domains. J. Lond. Math. Soc. 60, 237-257 (1999)

43. Savaré, G.: Regularity results for elliptic equations in lipschitz domains. J. Funct. Anal. 152(1), 176-201 (1998)

44. Schäffner, M.: Higher Integrability for Variational Integrals with Non-standard Growth. arXiv:2005.05115 (2020)

45. Schmidt, T.: Regularity of minimizers of $W^{1, p}$-quasiconvex variational integrals with (p, q)-growth. Calc. Var. 32, 1-24 (2008)

46. Schmidt, T.: Regularity theorems for degenerate quasiconvex energies with (p, q)-growth. Adv. Calc. Var. 1(3), 241-270 (2008)

47. Schmidt, T.: Regularity of relaxed minimizers of quasiconvex variational integrals with $(p, q)$-growth. Arch. Ration. Mech. Anal. 193, 311-337 (2009)

48. Tachikawa, A.: Boundary regularity of minimizers of double phase functionals. J. Math. Anal. Appl. $123946(2020)$

49. Triebel, H.: Interpolation Theory, Function Spaces, Differential Operators. VEB Deutscher Verlag der Wissenschaften, Berlin (1978)

50. Triebel, H.: Function spaces in Lipschitz domains and on Lipschitz manifolds. Characteristic functions as pointwise multipliers. Rev. Mathématica Complut. 15(2), 475-524 (2002)

51. Zhikov, V.V.: Averaging of functionals of the calculus of variations and elasticity theory. Izv. Math. 29(1), 33-66 (1987)

52. Zhikov, V.V.: Lavrentiev phenomenon and homogenization for some variational problems. C. R. Acad. Sci. Paris Sér. Mat. 50, 674-710 (1993)

53. Zhikov, V.V.: On Lavrentiev's phenomenon. Russ. J. Math. Phys. 3, 249-269 (1995)

Publisher's Note Springer Nature remains neutral with regard to jurisdictional claims in published maps and institutional affiliations. 